\title{
Adjectival and Adverbial Modification: The View from Modern Type Theories
}

\author{
Stergios Chatzikyriakidis ${ }^{1,2}$ - Zhaohui Luo $^{3}$
}

Published online: 17 February 2017

(C) The Author(s) 2017. This article is published with open access at Springerlink.com

\begin{abstract}
In this paper we present a study of adjectival/adverbial modification using modern type theories (MTTs), i.e. type theories within the tradition of Martin-Löf. We present an account of various issues concerning adjectival/adverbial modification and argue that MTTs can be used as an adequate language for interpreting NL semantics. MTTs are not only expressive enough to deal with a range of modification phenomena, but are furthermore well-suited to perform reasoning tasks that can be easily implemented (e.g. in proof-assistants) given their proof-theoretic nature. In MTT-semantics, common nouns are interpreted as types rather than predicates. Therefore, in order to capture the semantics of adjectives adequately, one needs to meet the challenge of modeling CNs modified by adjectives as types. To explicate that this can be done successfully, we first look at the mainstream classification of adjectives, i.e. intersective, subsective and non-subsective adjectives. There, we show that the rich type structure available in MTTs, along with a suitable subtyping framework, offers an adequate
\end{abstract}

Stergios Chatzikyriakidis: Partially supported by Centre for Linguistic Theory and Studies in Probability, University of Gothenburg.

Zhaohui Luo: Partially supported by EU COST Action CA15123 and CAS/SAFEA Inter. Partnership Program.

Stergios Chatzikyriakidis

stergios.chatzikyriakidis@gu.se

Zhaohui Luo

zhaohui.luo@hotmail.co.uk

1 Centre for Linguistic Theory and Studies in Probability, University of Gothenburg, Gothenburg, Sweden

2 Open University of Cyprus, Nicosia, Cyprus

3 Department of Computer Science, Royal Holloway, University of London, Egham, Surrey TW20 0EX, UK 
mechanism to model these cases. In particular, this modelling naturally takes care of the characterising inferences associated with each class of adjectives. Then, more advanced issues on adjectival modification are discussed: (a) degree adjectives, (b) comparatives and (c) multidimensional adjectives. There, it is shown that the use of indexed types can be usefully applied in order to deal with these cases. In the same vein, the issue of adverbial modification is discussed. We study two general typings for sentence and VP adverbs respectively. It is shown that the rich type structure in MTTs further provides useful organisational mechanisms in giving formal semantics for adverbs. In particular, we discuss the use of $\Sigma$-types to capture the veridicality/nonveridicality distinction and further discuss cases of intensional adverbs using the type theoretic notion of context (i.e. without resorting to intensional typing). We also look at manner, subject and speech act adverbials and propose solutions using MTTs. Finally, we show that the current proof technology can help mechanically check the associated inferences. A number of our proposals concerning adjectival and adverbial modification have been formalised in the proof assistant Coq and many of the associated inference patterns are checked to be correctly captured.

\section{Introduction}

The main subject of study of this paper is the modelling of adjectival and adverbial modification in doing semantics based on modern type theories (or MTT-semantics for short) (Ranta 1994; Luo 2012). It is well-known that adjectival and adverbial modification this a notoriously difficult issue to tackle adequately. The main reason behind this, is that the adjectival/adverbial classes are largely non-homogeneous semantic classes where a strict classification according to semantic properties is quite a difficult task. In MTT-semantics, common nouns are interpreted as types rather than predicates. This poses a new challenge: for example, the question of how to model CNs modified by various classes of adjectives as types in an adequate way. One of the aims of this paper is to take up these challenges; for example, we study how to model modified CNs by means of various type constructors in MTTs and hence show that MTTs provide adequate tools for this endeavour. Since MTTs have rich type structures, employing MTTs for formal semantics also provides us with various benefits that are not available in the Montagovian simple type-theoretic setting as we will argue in this paper. Furthermore, proof-theoretically defined, MTTs are the basis of various type theory based proof assistants which provide us the proof technology that can be used for computer-assisted reasoning based on MTT-semantics. We shall briefly illustrate this in terms of reasoning about modifications.

In this introduction, we shall first summarize the issues as regards adjectival and adverbial modification as discussed in the formal semantics literature, mostly within the Montagovian tradition. We want, in this respect, to exemplify the complex nature of the problem as well as to provide a background on the phenomena that we will attempt to account for. We shall then briefly discuss the challenge of modelling modification in MTT-semantics, where CNs are interpreted as types rather than predicates. 


\subsection{Adjectival and Adverbial Modifications: Some Summatory Notes}

Adjectival Modification Starting off with adjectives, a first coarse-grained distinction originating in earlier approaches within the Montagovian tradition (Montague 1973; Kamp 1975; Partee 2007, 2010), recognizes three main categories of adjectives: intersective, subsective and non-subsective (the latter involving two further subcategories), along with their respective associated inferences they give rise to:

(1) Intersective: $\operatorname{Adj}(N)(x) \Rightarrow \operatorname{Adj}(x) \wedge N(x)$

(2) Subsective: $\operatorname{Adj}(N)(x) \Rightarrow N(x)$

(3) Non-subsective (privative): $\operatorname{Adj}(N)(x) \Rightarrow \neg N(x)$

(4) Non-subsective (non-committal): $\operatorname{Adj}(N)(x) \Rightarrow$ ?

An example of an intersective adjective will be black. A black man for example is someone who is both black and a man. In a sense the blackness is not contingent on being a man. Thus, a black man is also a black human, a black animal and so on. To the contrary, subsective adjectives are contingent to the noun class they modify. Thus, a skilful surgeon is someone who is skilful as a surgeon, but we do not know if he is a skilful in general. Non-subsective adjectives on the other hand, involve two distinct categories: privative adjectives where the adjective-noun property entails the negation of the property of being a noun. Fake is a prototypical privative adjective. Lastly, non-commital adjectives involve adjectives that do not commit us to any of the aforementioned inferences. An example of such an adjective is alleged. An alleged thief might or might not be a thief.

All adjectives receive a unified intensional type from properties to properties, i.e. $(s \rightarrow(e \rightarrow t)) \rightarrow(s \rightarrow(e \rightarrow t)),{ }^{1}$ in Montagovian treatments (Kamp 1975; Partee 2007). Such a type can deal with intensional adjectives like former and alleged among others. This is indeed needed in order to avoid unwanted inferences like the following one shown below

\section{(5) John is an alleged thief $\Rightarrow$ John is a thief}

Intensional typing does the trick for cases like the one above. The main idea here is to generalize to the worst case, a technique frequently used in Montague semantics (the treatment of quantifiers being such a case). However, in order to take care of the inferences associated with the different types of adjectives, meaning postulates are introduced, which specify the exact semantics for each subclass. Such a postulate, the one for subsective adjectives, is shown below (modified from Partee 2007 ignoring intensional operators):

(6) $\forall Q: e \rightarrow t \cdot \forall x: e \cdot A D J(Q, x) \supset Q(x)$

Generalizing to the worst case, in the case of adjectives the use of intensional typing across the board, i.e also for adjectives (as well as adverbs) that are not intensional,

\footnotetext{
1 Within the simple type theory used in Montague Grammar, $e$ is the type of individuals, $t$ is the type of truth-values and $s$ the type of world-time pairs.
} 
is counterintuitive. Furthermore, the use of meaning postulates will be shown not to be needed, at least for intersective and subsective adjectives, if one moves to a rich type theory with subtyping, like the one we use in this paper. This has been already exemplified in Chatzikyriakidis and Luo (2013) and it is going to be discussed in this paper as well.

Now, anyone who has looked at the semantics of adjectives in more detail knows that the classification into intersective, subsective and non-subsective adjectives, even though a useful one, is rather coarse-grained for a number of cases. In order to have a deeper understanding of the semantics of adjectives (or aspects of their semantics to be more precise), a number of further issues that require a more fine-grained semantic treatment need be taken care of. One issue at hand involves gradable adjectives. Gradable adjectives have been traditionally treated as involving an extra parameter, that of a DEGREE variable that can be bound by different morphosyntactic forms of the adjective, i.e. its comparative (see Von Stechow 1984; Heim 2000 among others). One standard way of looking at this extra complication, is that gradable adjectives involve some kind of measurement. Usually, this measurement is taken to be a degree argument. Thus, the presence of a degree argument is then taken to be the main difference between gradable and non-gradable adjectives. This degree argument has been proposed in the literature to be either formally encoded in the typing as in Bartsch and Vennemann (1973), Von Stechow (1984), Heim (2000), or not as in Lewis (1970); McConnell-Ginet (1973), Klein (1980), van Benthem (2012). To give an example, the adjective small, will involve the following definition according to the first view: ${ }^{2}$

(7) $\llbracket$ small $\rrbracket=\lambda d:$ Degree. $\lambda x$ :e.Height $(x) \leq d$

Another issue concerning adjectives is the way the positive form is connected with the comparative as well as the superlative form.

Lastly, there are a number of adjectives, the so-called multidimensional adjectives that present added complications; their meanings do not just involve one dimension (e.g. the dimension of height in the case of tall), but more than one. Classical cases involve adjectives like healthy and sick or even adjectives like big. The intuition is that adjectives like e.g. healthy quantify over a number of dimensions, e.g. blood pressure, cholesterol etc. (Sassoon 2012). Similarly, big may involve different dimensions like height, width etc. Cases of multidimensional adjectives rely heavily on the context with respect to the dimensions that need to be satisfied in order for an utterance to be felicitous. The insight provided by Sassoon (2012) is that there are two classes of multidimensional adjectives, positive and negative. Positive adjectives incude cases like healthy, while negative ones cases like sick. The difference in each case concerns the form of quantification over dimensions in each case, universal quantification over

\footnotetext{
${ }^{2}$ In this paper, we shall use the notation $\llbracket w \rrbracket$ for the semantics of $w$. For example, for the CN human,

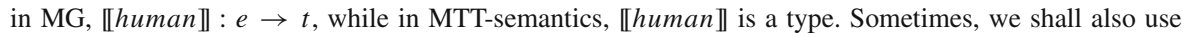
capitalised words for types in MTTs: for example, we might use Human for the type of humans; in this case, «human $\rrbracket$ and Human are the same types.
} 
dimensions for positive multidimensional adjectives, existential quantification over dimensions for negative ones. Consider the following examples:

(8) Dan is healthy except with respect to blood pressure

(9) \# Dan is sick except with respect to blood pressure

Examples like above exemplify the latter claim. In this sense, the two classes of adjectives have also different inferential properties. A sentence like John is healthy implies that John is healthy with respect to all "health" dimensions while in a sentence like John is sick, what we get is an entailment that John is not healthy across some dimension.

Adverbial Modification Adverbials, similarly to adjectives, are a largely nonhomogeneous class. Given this observation, it is not surprising that various classifications have been proposed throughout the years. According to one of the most prominent ones, that of Ernst (2002) and Maienborn and Schafer (2011), adverbs receive a tripartite classification with further subclassifications for each class: (a) predicational, (b) participant oriented and (c) functional adverbials. ${ }^{3}$

Predicational adverbs comprise the main bulk of adverbs. Its main subcategories including sentence and verb related adverbs. Sentence adverbs are further classified into subject oriented adverbs like arrogantly, speaker oriented adverbs that include speech act adverbials like honestly, epistemic adverbials like possibly and domain adverbs like botanically, while verb related adverbials into mental attitude adverbs like reluctantly, manner adverbs like skillfully and degree adverbs like deeply. Participant oriented adverbials on the other hand include adverbials that introduce a new entity/entities to the situation/event described by the proposition in question. Examples of this type of adverbials include cases like with a knife, with a gun etc. Lastly, functional adverbials include adverbials where some kind of quantification is involved like usually, never etc.

The main bulk of proposals as regards adverbials falls within two lines of approach: (a) the operator approach proposed within the Montagovian tradition and (b) the (neo)Davidsonian event-related approach. Both ways of looking at the issue have their merits as well as their disadvantages. The operator approach (see Montague 1970; Thomason and Stalkaner 1973; Kamp 1975 among others), views adverbs as functors which return the same type as that of their argument. Furthermore, the operator approach distinguishes between adverbs that take a truth value (or a proposition) to return a truth value (or proposition) and adverbs that take a set (or concept) to return a set (or concept):

(10) Extensional: $(e \rightarrow t) \rightarrow(e \rightarrow t)$

Intensional: $(s \rightarrow(e \rightarrow t)) \rightarrow(s \rightarrow(e \rightarrow t))$

(11) Extensional: $t \rightarrow t$

Intensional: $(s \rightarrow t) \rightarrow(s \rightarrow t)$

\footnotetext{
3 Maienborn and Schafer (2011) uses only semantic criteria, while Ernst (2002) mostly concentrates on the syntax of adverbs. See the individual pieces of work for more details.
} 
The above typings correspond to sentence adverbs (e.g. evaluative adverbs like fortunately) and VP adverbs (e.g. manner adverbs). Their intensionalized typing versions make the rather welcome prediction that in VP-adverbs, opaque contexts should arise for the object but not for the subject, a prediction which is borne out from the facts (see Kamp 1975; Thomason and Stalkaner 1973; Maienborn and Schafer 2011 for a summary). To give an example: imagine a VP-adverb like intentionally in a sentence like Oedipus intentionally married Jocaste. Under the intensionalized VP-adverb typing, we have that Oedipus intentionally married his mother does not follow. However, it does follow that the son of Laius intentionally married Jocaste. Similarly, it correctly predicts that for sentence intensional adverbs, opacity should arise for both the subject and the object. Thus, in a sentence like Oedipus allegedly married Jocaste, both the son of Laius allegedly married Jocaste and Oedipus allegedly married his mother do not follow. On the other hand, approaches within the tradition initiated by Davidson (1967), argue that adverbs can be seen as providing restrictions w.r.t. the event denoted by the sentence/proposition in each case. In effect, adverbs in these approaches are assumed to modify the event in some way. For example a manner adverb like beautifully is taken to involve event modification. This is general embedded within a theory which takes the semantics of semantics to boil down to a conjunction of predicates applied to either entity or event arguments. Consider the sentence below:

\section{(12) Mary dances beautifully}

The classic analysis of manner adverbs within the Neo-Davidsonian tradition is one where manner adverbs are seen as modifiers over events. Informally the sentence means that there is a dancing event, of which Mary is the agent, and this event is a beautiful one. Somewhat more formally:

\section{$\exists e[\operatorname{dancing}(\operatorname{Mary}, e) \wedge$ beautiful $(e)])$}

The problem here, as already noted in the literature, is that the manner of the event and not the event itself is modified. Thus, one might want to introduce manner in the ontology of types/basic predicates (depending on whether we have a typed or a non-typed system). We believe that this extension can be done very easily within a rich type system as the one we are endorsing in this paper.

Other types of adverbials that we are going to be looking in this paper, include subject oriented and and agent oriented adverbs. In the former case, we find adjectives like stupidly, where in this case not only a manner of the event is denoted but also the agent of the event:

(14) John stupidly opened the door $\Rightarrow$ the manner that John opened the door was stupid

Agent oriented adverbs on the other hand, seem to provide commentary with respect to the utterance. An adverb like frankly seems to imply that a sentence of the form frankly P means something like "I frankly tell you that P". We will try to show, as in the case of manner adverbs, that the elaborate typing mechanisms of MTTs can be 
used in order to get this fine-grained meaning nuances associated with the different kinds of adverbs.

\subsection{Adjectival/Adverbial Modifications in MTT-Semantics}

The major aim of the paper is to study how to deal with adjectival and adverbial modification in MTT-semantics. As briefly mentioned previously, in order to model adjectival modification adequately in MTT-semantics, one has to meet the challenge of how to interpret CNs modified by various classes of adjectives as types rather than predicates.

As we know, in foundational languages of formal semantics, types in type theories are different from sets in set theory, although both represent collections of objects/elements. In a nutshell and very informally, the difference may be summarised by saying that such types are only manageable sets in the sense that some sets and set operations (e.g. intersection and union), are not available in the world of types for, otherwise, some of the salient and important properties of such type theories would be lost (see, for example, Pierce 1991 for more information). For example, in type theories for formal semantics (either the simple type theory STT as used in Montague's semantics or MTTs in MTT-semantics), type checking is decidable; in layman's terms, it is mechanically checkable whether any object a is of type A. In contrast, the truth of the membership relation $s \in S$ in set theory is undecidable since it is just a logical formula in first-order logic. In STT, this means that one can check mechanically whether an object is of type e of entities, or of type t of propositions, or of a function type $A \rightarrow B$. For STT, obviously this must be decidable for otherwise the internal higher-order logic would not work properly (e.g. the applications of its rules would become infeasible). This is similarly the case for MTTs for otherwise one would not have a working internal logic that is necessary for formal semantics. Furthermore, these properties have other advantages: for example, given that MTTs are proof-theoretically specified, they can be effectively implemented on computers - the systems called proof assistants. When formal semantics are given using MTTs, they can be directly implemented in a proof assistant which, among other things, provides a tool for natural language inference based on the implemented MTT-semantics.

Interpreting $\mathrm{CNs}$ as types, one faces the challenge that there should be enough types to interpret various classes of CNs adequately. This is a difficult challenge because, even in MTTs which have many inductively defined types, there are less types than predicates/sets. In this paper, we shall show that various type constructors in MTTs provide us with adequate mechanisms to model adjectival/adverbial modification of various kinds. For instance, we will show how to employ $\Sigma$-types for intersective adjectives, disjoint union types for privative adjectives, and polymorphic $\Pi$-types with universes and $\Sigma$ types for subsective adjectives. ${ }^{4}$ In general, we shall illustrate that, using the rich type structure of MTTs, we can provide an account of a number of issues in adjectival/adverbial modification.

\footnotetext{
${ }^{4}$ See next section for an the explanation of these types.
} 
It may be useful to emphasise that the scope of the current paper is a rather moderate one. Our aim, as already mentioned, is to show the way modification can be treated in a framework where CNs are modelled as types instead of predicates (or funcational sets of type $e \rightarrow t$ ). As such, this paper should not be taken as a paper that tries to compete with the other state-of-the-art approaches found in the considerably rich formal semantics literature in all the aspects that this paper discusses. This is a paper that aspires to show the way to use an alternative formal semantics theory for the study of linguistic semantics. On the other hand, we do not want this paper to be seen as framework gymnastics, i.e. a plain exercise in formal semantics using just another framework. MTTs, as already mentioned, have a number of advantages compared to the simple type theory when it comes to their respective computational properties as well as their fitness to support proof-theoretic reasoning (cf., decidability and practical inference in proof assistants, as mentioned above). These two latter properties of MTTsemantics and their potential application in the study of natural language inference not only from a semantic but from a computational point of view, provide additional reasons for us to believe that the ideas put forth in this paper are worth pursuing.

In Sect. 2, we introduce the core features of modern type theories, emphasizing those relevant to this paper, setting up the background knowledge and notation. In Sects. 3 and 4 we deal with a number of aspects of adjectival modification: (a) the traditional classification into intersective, subsective and non-subsective adjectives in Sect. 3, and (b) gradable and multidimensional adjectives in Sect. 4. In Sect. 5, we look at adverbial modification. There, building on work by Chatzikyriakidis (2014), we show how the rich typing constructs of MTTs can give us a way out with respect to veridicality, intensional adverbs and some aspects concerning manner and X-oriented adverbs. Lastly, in Sect. 6, we check some of the proposals in this paper in terms of their inferential properties. We show how MTTs can be used in this respect both from a theoretical as well as an implementational point of view. With respect to the latter, we implement some of the ideas in this paper in the Coq proof assistant (Coq 2007), showing its potential use to the study of NL inference.

\section{MTTs with Coercive Subtyping: Introduction}

In this section, we give a brief introduction to formal semantics based on modern type theories (MTTs) (Ranta 1994; Luo 2010, 2012). We will try and introduce MTTs by exemplifying its various features related to linguistic semantics. A modern type theory is a variant of a class of type theories as studied by Martin-Löf $(1975,1984)$ and others, which have dependent types and inductive types, among others. Among MTTs, we are going to employ the Unified Theory of dependent Types (UTT) (Luo 1994) with the addition of the coercive subtyping mechanism (see, for example, Luo 1999; Luo et al. 2012 and below).

\subsection{Type Many-Sortedness and CNs as Types}

A difference between MTT-semantics and Montague semantics lies in the interpretation of common nouns (CNs). In Montague (1974) semantics, the underlying logic 
(Church's 1940 simple type theory) can be seen as 'single-sorted' in the sense that there is only one type $e$ of all entities. The other types such as $t$ of truth values and the function types generated from $e$ and $t$ do not stand for types of entities. In this respect, there are no fine-grained distinctions between the elements of type $e$ and as such, all individuals are interpreted using the same type. For example, John and Mary have the same type in simple type theories, the type $e$ of individuals. An MTT, on the other hand, can be regarded as a 'many-sorted' logical system in that it contains many types. In this respect, in MTT-semantics one can make fine-grained distinctions between individuals and use those different types to interpret subclasses of individuals.

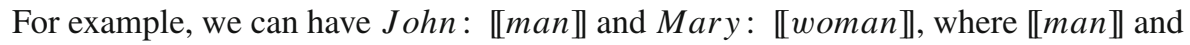

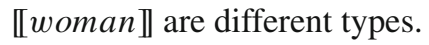

An important trait of MTT-semantics is the interpretation of CNs as types (Ranta 1994) rather than sets or predicates (i.e. objects of type $e \rightarrow t$ ) as in Montague

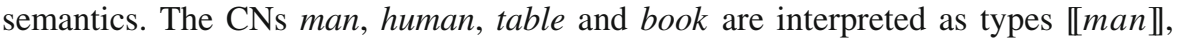
$\llbracket h u m a n \rrbracket, \llbracket t a b l e \rrbracket$ and $\llbracket b o o k \rrbracket$, respectively. Then, individuals are interpreted as being of one of the types used to interpret CNs.

This many-sortedness (i.e. the fact that there are many basic types in an MTT, but also typing constructors like $\Sigma, \Pi$ ) has the welcome result that a number of semantically infelicitous sentences like e.g. the ham sandwich walks, which are however syntactically well-formed, can be explained easily given that a verb like walks will be specified as being of type Animal $\rightarrow$ Prop, while the type for ham sandwich will be

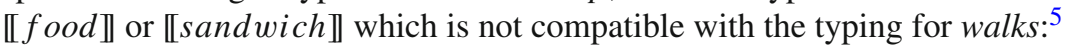

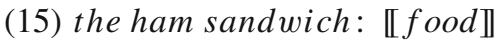

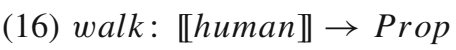

The idea of common nouns being interpreted as types rather than predicates has been argued in Luo (2012a) on philosophical grounds as well. There, the second author argues that Geach's observation that common nouns, in contrast to other linguistic categories, have criteria of identity that enable common nouns to be compared, counted or quantified, has an interesting link with the constructive notion of set/type: in constructive mathematics, sets (types) are not constructed only by specifying their objects but they additionally involve an equality relation. The argument is then that the interpretation of CNs as types in MTTs is explained and justified to a certain extent. ${ }^{6}$

Interpreting $\mathrm{CNs}$ as types rather than predicates has also a significant methodological implication: this is compatible with various subtyping relations one may consider in formal semantics. For instance, in modelling some linguistic phenomena semantically, one may introduce various subtyping relations by postulating a collection of subtypes (physical objects, informational objects, eventualities, etc.) of the type of entities (Asher 2012). It has become clear that, if CNs are interpreted as predicates as in the traditional Montagovian setting, introducing such subtyping relations would cause problems: even some basic semantic interpretations would go wrong and it is

\footnotetext{
5 This is of course based on the assumption that the definite NP is of a lower type and not a Generalized Quantifier.

6 See Luo (2012a) for more details on this.
} 
very difficult to deal with some linguistic phenomena such as copredication satisfactorily. Instead, if CNs are interpreted as types, as in MTTs, copredication can be given a straightforward and satisfactory treatment (Luo 2010).

$\Sigma$-types, П-types, indexed types and Universes We shall introduce several dependent types and the notion of type universe.

Dependent $\Sigma$-types. One of the basic features of MTTs is the use of Dependent Types. A dependent type is a family of types that depend on some values. The constructor/operator $\Sigma$ is a generalization of the Cartesian product of two sets that allows

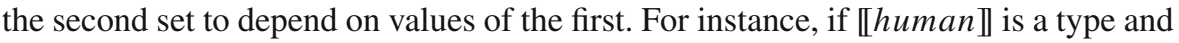

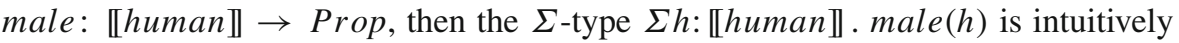
the type of humans who are male.

More formally, if $A$ is a type and $B$ is an $A$-indexed family of types, then $\Sigma(A, B)$, or sometimes written as $\Sigma x: A . B(x)$, is a type, consisting of pairs $(a, b)$ such that $a$ is of type $A$ and $b$ is of type $B(a)$. When $B(x)$ is a constant type (i.e. always the same type no matter what $x$ is), the $\Sigma$-type degenerates into product type $A \times B$ of nondependent pairs. $\Sigma$-types (and product types) are associated projection operations $\pi_{1}$ and $\pi_{2}$ so that $\pi_{1}(a, b)=a$ and $\pi_{2}(a, b)=b$, for every $(a, b)$ of type $\Sigma(A, B)$ or $A \times B$.

The linguistic relevance of $\Sigma$-types can be directly appreciated once we understand that in its dependent case, $\Sigma$-types can be used to interpret linguistic phenomena of central importance, like adjectival modification (see above for interpretation of modified CNs) (Ranta 1994). For example, handsome man is interpreted as $\Sigma$-type (17), the type of handsome men (or more precisely, of those men together with proofs that they are handsome):

\section{(17) $\Sigma m: \llbracket m a n \rrbracket . \llbracket h a n d s o m e \rrbracket(m)$}

where 【handsome $\rrbracket(m)$ is a family of propositions/types that depends on the man $m$.

Dependent $\Pi$-types The other basic constructor for dependent types is $\Pi$. $\Pi$-types can be seen as a generalization of the normal function space where the second type is a family of types that might be dependent on the values of the first. A $\Pi$-type degenerates to the function type $A \rightarrow B$ in the non-dependent case. In more detail, when $A$ is a type and $P$ is a predicate over $A, \Pi x: A . P(x)$ is the dependent function type that, in the embedded logic, stands for the universally quantified proposition $\forall x: A . P(x)$. For example, the following sentence (18) is interpreted as (32):

(18)Every man walks.

(19) $\Pi x: \llbracket m a n \rrbracket \cdot \llbracket w a l k \rrbracket(x)$

$\Pi$-types are very useful in formulating the typings for a number of linguistic categories like VP adverbs or quantifiers. The idea is that adverbs and quantifiers range over the universe of (the interpretations of) CNs and as such we need a way to represent this fact. In this case, $\Pi$-types can be used, universally quantifying over the universe $\mathrm{CN}$. (20) is the type for VP adverbs ${ }^{7}$ while (21) is the type for quantifiers:

\footnotetext{
7 This was proposed for the first time in Luo (2011b).
} 
(20) $\Pi$ A: CN. $(A \rightarrow$ Prop $) \rightarrow(A \rightarrow$ Prop $)$

(21) $\Pi$ A: CN. $(A \rightarrow$ Prop $) \rightarrow$ Prop

Further explanations of the above types are given after we have introduced the concept of type universe below.

Indexed types Indexed types are special kinds of dependent types where the type depends on an index. In effect we are dealing with families of types that are indexed by a type parameter. The type parameter is usually a simple one in most cases. Examples of indices include, for instance, the type $N$ of natural numbers, the type Human of human beings, and the type Height of heights (see below). We can think for example that for $h$ :Human, there is a family of types $E v t(h)$ of events that are conducted by $h$. In the same sense, one can also think that for $h$ :Height, there is a family of types $\operatorname{Human}(h)$ of humans with a height parameter. This types will be of great importance in our discussion of gradable adjectives in Sect. 4.

Type Universes An advanced feature of MTTs, which will be shown to be very relevant in interpreting NL semantics in general as well as adjectival modification specifically, is that of universes. Informally, a universe is a collection of (the names of) types put into a type (Martin-Löf 1984). ${ }^{8}$ For example, one may want to collect all the names of the types that interpret common nouns into a universe $\mathrm{CN}$ : Type. The idea is that for each type $A$ that interprets a common noun, there is a name $\bar{A}$ in $\mathrm{CN}$. For example,

\section{$\overline{\llbracket m a n \rrbracket}: \mathrm{CN}$ and $T_{\mathrm{CN}}(\overline{\llbracket m a n \rrbracket})=\llbracket m a n \rrbracket$.}

In practice, we do not distinguish a type in $\mathrm{CN}$ and its name by omitting the overlines and the operator $T_{\mathrm{CN}}$ by simply writing, for instance, $\llbracket m a n \rrbracket: \mathrm{CN}$. Thus, the universe includes the collection of the names that interpret common nouns. For example, in CN, we shall find the following types:

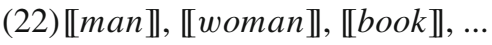

(23) $\Sigma m: \llbracket m a n \rrbracket . \llbracket h a n d s o m e \rrbracket(m)$

(24) $G_{R}+G_{F}$

where the $\Sigma$-type in (23) is the proposed interpretation of handsome man and the disjoint union type in (24) is that of 'gun' (the disjoint union of real guns and fake guns - see the discussion in Sect. 3). We can furthermore use partitions of the universe $\mathrm{CN}$ that would correspond to more restrictive universes that might be needed by different predicates. For example, one might assume predicates having a restriction which only allows arguments that are of type Human or any of its subtypes. In that

\footnotetext{
8 There is quite a long discussion on how these universes should be like. In particular, the debate is largely concentrated on whether a universe should be predicative or impredicative. A strongly impredicative universe $U$ of all types (with $U: U$ and $\Pi$-types) is shown to be paradoxical (Girard 1971) and as such logically inconsistent. The theory UTT we use here has only one impredicative universe Prop (representing the world of logical formulas) together with an infinitely many predicative universes which as such avoids Girard's paradox (see Luo 1994 for more details).
} 


$$
\overline{\text { Human }: \mathrm{CN}_{H}} \quad \frac{A<\text { Human }}{A: \mathrm{CN}_{H}} \quad \frac{A: \mathrm{CN}_{H}}{A: \mathrm{CN}}
$$

Fig. 1 Introduction rules for $\mathrm{CN}_{H}$

case, one can introduce the subuniverse $\mathrm{CN}_{H}$ that consists of type Human and all its sybtypes (Fig. 1). It goes without saying that the universe $\mathrm{CN}$ is an open universe, where additional types can be always added.

Quantifiers and adverb typing Having introduced the universe CN, it is now possible to explain (20) and (21). The difference between this type and the type of GQs in Montague Grammar is that in the latter case we have a relation between two sets, i.e. two predicates of type $e \rightarrow t$. The type for quantifiers is shown below (ignoring intensions):

$(25)(e \rightarrow t) \rightarrow(e \rightarrow t) \rightarrow t$

The first predicate is the type for the noun, given that nouns are considered to be predicates in MG, and the second predicate is the one denoted by the verb. In MTT semantics where common nouns are types and not predicates, the relation is now between a type and a predicate. Typing has to further be polymorphic given that we have a multitude of basic types, and in principle we would want to have quantification with all these types. The solution is to have a polymorphic type extending over the universe $\mathrm{CN}$, i.e. the type in (20). Thus, we first need an argument of type $A, A: \mathrm{CN}$. This corresponds to the $\mathrm{CN}$ argument. As soon as we get this argument, what we get back is the type $(A \rightarrow$ Prop $) \rightarrow$ Prop. Type polymorphy will predict that the type returned will be dependent on the value of $A$. If $A=\llbracket m a n \rrbracket$ then the type returned will be $(\llbracket m a n \rrbracket \rightarrow$ Prop $) \rightarrow$ Prop , if $A=\llbracket$ human $\rrbracket,(\llbracket$ human $\rrbracket \rightarrow$ Prop $) \rightarrow$ Prop and so on. For example, some human is of type (【human $\rightarrow \rightarrow$ Prop $) \rightarrow$ Prop given

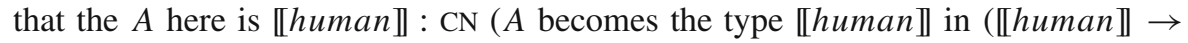

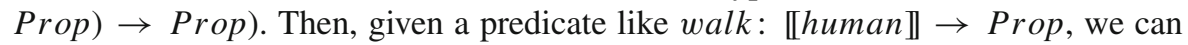

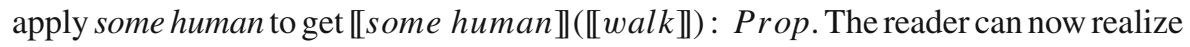
how the adverb typing in (25) is to be understood.

Subtyping in Formal Semantics As briefly explained above, given the many-sortedness of MTTs, CNs can be interpreted as types. For instance, in a Montagovian setting, all of the verbs below are given the same type $(e \rightarrow t)$, but in an MTT, we can have:

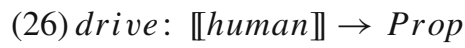

(27) eat : 【animal $\rightarrow$ Prop

(28) disappear : 【object $\rightarrow$ Prop

which have different domain types. This has the advantage of disallowing interpretations of infelicitous examples like the ham sandwich walks.

However, interpreting CNs by means of different types could lead to serious undergeneralizations without a subtyping mechanism. Thus, subtyping is crucial for MTT-semantics. For instance, consider the interpretation of the sentence a man talks 


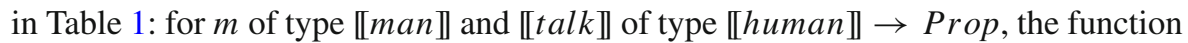
application $\llbracket \operatorname{tal} \rrbracket \rrbracket(m)$ is only well-typed because we assume $\llbracket m a n \rrbracket$ be a subtype of

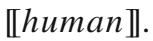

Coercive subtyping (Luo 1999; Luo et al. 2012) provides an adequate framework to be employed for MTT-based formal semantics (Luo 2010, 2012). ${ }^{9}$ It can be seen as an abbreviation mechanism: $A$ is a (proper) subtype of $B(A<B)$ if there is a unique implicit coercion $c$ from type $A$ to type $B$ and, if so, an object $a$ of type $A$ can be used in any context $\mathfrak{C}_{B}\left[\_\right]$that expects an object of type $B: \mathfrak{C}_{B}[a]$ is legal (well-typed) and equal to $\mathfrak{C}_{B}[c(a)]$.

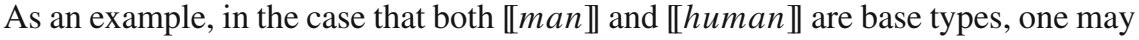
introduce the following as a basic subtyping relation:

\section{$\llbracket m a n \rrbracket<\llbracket h u m a n \rrbracket$}

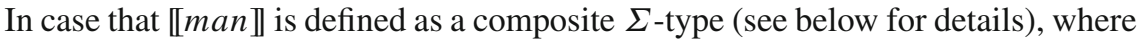

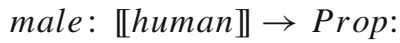

(30) $\operatorname{man} \rrbracket=\Sigma h: \llbracket h u m a n \rrbracket . \operatorname{male}(h)$

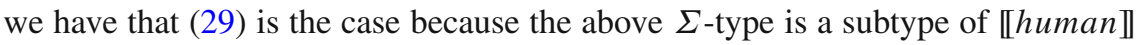
via the first projection $\pi_{1}$ :

\section{(31) $\Sigma h: \llbracket h u m a n \rrbracket . m a l e(h)<_{\pi_{1}} \llbracket$ human $\rrbracket$}

Equipped with this coercive subtyping mechanism, the undergeneration problems can be straightforwardly solved while still retaining the ability to rule out semantically infelicitous cases like the ham sandwich walks. In effect, many-sortedness in MTTs turns out to be superior than single sortedness in simple type theory (at least in this respect). Furthermore, many inferences concerning the monotonicity on the first argument of generalized quantifiers can be directly captured using the subtyping mechanism. In effect an inference of the sort exemplified in the example (32) below, can be captured given that $\llbracket m a n \rrbracket<\llbracket h u m a n \rrbracket:$

\section{(32) Some man runs $\Rightarrow$ Some human runs}

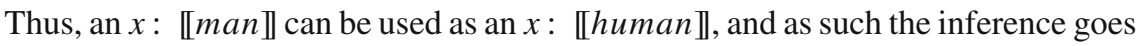
through for 'free' in a way.

Another important trait of the coercive subtyping mechanism, which will be very important in our discussion of intersective adjectives, is that subtyping also propagates

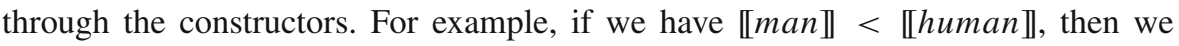

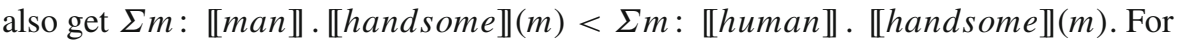
more information on subtyping propagation see, for example, Luo (1999).

\footnotetext{
${ }^{9}$ It is worth mentioning that subsumptive subtyping, the traditional notion of subtyping that adopts the subsumption rule (if $A \leq B$, then every object of type $A$ is also of type $B$ ), is inadequate for MTTs in the sense that it would destroy some important properties of MTTs [see, for example, Section 4 of Luo et al. (2012) for details].
} 
Coercion Contexts and Local Coercions It is a well-known fact that word meanings heavily rely on contexts. In this sense, a lot of the times we need to deal with cases like the following classic meaning transfer example from (Nunberg 1995):

Example 1 (reference transfer) Consider the following utterance (cf., Nunberg 1995):

(33) The ham sandwich shouts.

Assuming that the act of shouting requires that the argument be human, it is obvious that sentence (33) is not well-formed, unless it is uttered by somebody in some special extralinguistic context (e.g. by a waiter in a café to refer to a person who has ordered a ham sandwich).

This kind of local coercions or local subtypes can be dealt with in an MTT via using the type theoretical notion of context. A context is of the following form:

$$
x_{1}: A_{1}, \ldots, x_{n}: A_{n}
$$

where $A_{i}$ is can be a data type, in which case $x_{i}$ is assumed to be an object of that type, or a logical proposition, in which case the proposition $A_{i}$ is assumed to be true and $x_{i}$ a proof of $A_{i}$. For example, one may have the following context:

$$
m: \llbracket m a n \rrbracket, h p r o o f: \llbracket \text { handsome }(m)
$$

basically an assumption that $m$ is of type man ( $m$ is a man) and a proof that this $m$ is handsome. The context can furthermore be extended to involve subtyping declarations of the following form:

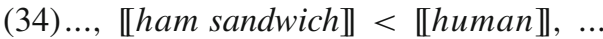

where what we have is that type ham is coerced into human in this particular setting. The range of coercions we can perform can get more-fine grained as it has been exemplified for example in the work of Asher and Luo (2012). The interested reader is referred there for more information on this issue. People interested in seeing the use of contexts for NL semantics, please see Ranta (1994), Boldini (2001) as well as Chatzikyriakidis and Luo (2014c) for the similar notion of signature.

Proof-theoretic Facet of MTT-semantics One of the key features of formal semantics in MTTs is its proof-theoretic facet. It has been pointed out in Luo (2014) that MTT-semantics is both model-theoretic and proof-theoretic. Without getting into the details, we emphasise here the proof-theoretic characteristics of MTT-semantics: it allows us to understand MTT-semantics in a proof-theoretic way [as logics can be understood proof-theoretically (Kahle and Schroeder-Heister 2006)] and, furthermore, allows a direct application of type theory based proof assistants such as Coq (2007) in conducting practical inferences based on MTT-semantics. This has amounted to a computational treatment of formal semantics and opens up a new avenue in semanticsbased reasoning in natural language (see Chatzikyriakidis and Luo 2014b and Sect. 6). 
Table 1 Examples in formal semantics

\begin{tabular}{|c|c|c|c|}
\hline & Example & Montague semantics & MTT-semantics \\
\hline $\mathrm{CN}$ & Man, human & $\llbracket m a n \rrbracket, \llbracket$ human $\rrbracket: e \rightarrow t$ & $\llbracket m a n \rrbracket, \llbracket h u m a n \rrbracket:$ Type \\
\hline IV & Talk & $\llbracket t a l k \rrbracket: e \rightarrow t$ & $\llbracket$ talk $\rrbracket: \llbracket h u m a n \rrbracket \rightarrow$ Prop \\
\hline ADJ & Handsome & $\begin{array}{l}\text { 【handsome } \rrbracket: \\
\qquad(e \rightarrow t) \rightarrow(e \rightarrow t)\end{array}$ & $\llbracket$ handsome $\rrbracket: \llbracket m a n \rrbracket \rightarrow$ Prop \\
\hline $\mathrm{ADV}_{V P}$ & Quickly & 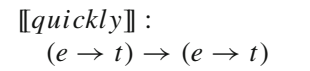 & $\begin{array}{r}\llbracket q u i c k l y \rrbracket: \Pi A: \mathrm{CN} .(A \rightarrow \\
\text { Prop }) \rightarrow(A \rightarrow \text { Prop })\end{array}$ \\
\hline Quantifier & Some & $\begin{array}{l}\llbracket \text { some } \rrbracket:(e \rightarrow t) \\
\quad \rightarrow(e \rightarrow t) \rightarrow t\end{array}$ & $\begin{array}{c}\llbracket \text { some } \rrbracket: \Pi A: \mathrm{CN} .(A \rightarrow \\
\text { Prop }) \rightarrow \text { Prop }\end{array}$ \\
\hline $\mathrm{MCN}$ & Handsome man & $\begin{array}{l}\llbracket \text { \handsome } \rrbracket(\llbracket \operatorname{man} \rrbracket): \\
\quad e \rightarrow t\end{array}$ & $\begin{array}{l}\Sigma m: \llbracket m a n \rrbracket . \llbracket \text { handsome } \rrbracket(m) \\
\quad: \text { Type }\end{array}$ \\
\hline $\mathrm{S}$ & A man talks & $\begin{array}{l}\exists m: e . \llbracket m a n \rrbracket(m) \\
\quad \& \llbracket \operatorname{tal} \| \rrbracket(m): t\end{array}$ & $\exists m: \llbracket m a n \rrbracket . \llbracket \operatorname{talk} \rrbracket(m): \operatorname{Prop}$ \\
\hline
\end{tabular}

Discussing this is out of the scope of the current paper. The interested reader may consider reading some related papers including, for example, Luo (2014).

MTT semantics interpreting natural language semantics: Preliminaries Recapitulating somehow from the previous discussion, we present the way basic linguistic categories are interpreted in MTT-semantics:

- A sentence $(\mathrm{S})$ is interpreted as a proposition of type Prop.

- A common noun $(\mathrm{CN})$ can be interpreted as a type.

- A verb (IV) can be interpreted as a predicate over the type $D$ that interprets the domain of the verb (ie, a function of type $D \rightarrow$ Prop).

- An adjective (ADJ) can be interpreted as a predicate over the type that interprets

- A VP adverb can be interpreted as a function from predicates $(A \rightarrow$ Prop $)$ to predicates $(A \rightarrow$ Prop) where the $A$ extends over the universe $\mathrm{CN}$

- A quantifier is interpreted as a function from a type $A$ :CN extending over the universe $\mathrm{CN}$ to a function from predicates over $A$ to propositions. ${ }^{10}$ the domain of the adjective (ie, a function of type $D \rightarrow$ Prop).

- Modified common nouns (MCNs) can be interpreted by means of $\Sigma$-types (see below).

Basic examples are shown in Table 1, along with a comparison with their counterparts in Montague semantics.

Note that this table shows some basic comparisons to Montague semantics as well as potential ways to interpret these categories. For some of these cases, other ways of interpretation will be pursued. For example, besides the use of a basic predicate type for adjectives, type polymorphy will be used for e.g. cases of subsective adjectives.

\section{Intersective, Subsective and Non-subsective Adjectives}

In this section, we look at the traditional formal semantics classification of adjectives (Kamp 1975; Kamp and Partee 1995; Partee 2007) and discuss the solutions using

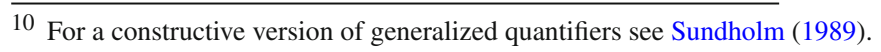


MTTs based on earlier work of ours (Chatzikyriakidis and Luo 2013). Historically, Ranta (1994) was the first to propose the use of $\Sigma$-types to represent adjectival modification. ${ }^{11}$ However, the proposal was not completely working because there was no proper subtyping mechanism that is essential in order for $\Sigma$-types to be employed for adjectival modification. ${ }^{12}$ This problem was solved in Luo (2010), where the second author proposed to employ coercive subtyping (Luo 1999; Luo et al. 2012). As a consequence, $\mathrm{CNs}$ modified by intersective adjectives can be properly represented by means of $\Sigma$-types.

Subsective adjectival modification was then studied in Chatzikyriakidis and Luo (2013) where the authors proposed to use the universe CN of common nouns and polymorphism in representations of subjective adjectives like small,large. Non-subsective adjectives have also been studied: for privative adjectives like fake, (Luo 2011a; Chatzikyriakidis and Luo 2013) propose to use disjoint union types while, for noncommittal adjectives like alleged, belief contexts (Chatzikyriakidis and Luo 2013). All these proposed solutions maintain a lower type for adjectives. The rich typing provided by MTTs in conjunction with coercive subtyping (Luo 1999; Luo et al. 2012) can give us an attractive solution to all adjectives under the traditional classification into intersective, subsective and non-subsective adjectives.

\subsection{Intersective and Subsective Adjectives}

Intersective and subsective adjectives can be treated properly using $\Sigma$-types. Using $\Sigma$-types to represent adjectival modification, as already mentioned, was originally proposed by Ranta (1994). However, in Ranta's account, neither of the two classes can be captured successfully: for intersective adjectives, we lack a proper subtyping mechanism and, for subsective adjectives, we need to use the universe of common nouns and polymorphism.

The $\Sigma$-type treatment is straightforward. In MTT-semantics, CNs are interpreted as types and adjectives as predicates, i.e. functions of type $A \rightarrow \operatorname{Prop}$, for some type $A$ : $C N$. Thus, an adjective like black (on the assumption that black is intersective) will be of type $\llbracket$ object $\rrbracket \rightarrow$ Prop, an adjective like handsome of type $\llbracket$ human $\rrbracket \rightarrow$ Prop ,

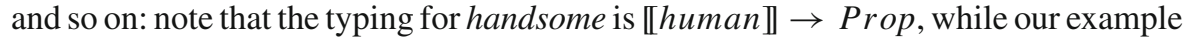
involves the type man. The reason this example is well-formed, as already discussed in the introduction to MTT semantics, is due to the subtyping relation man < human and the fact that subtyping propagates through the constructors.

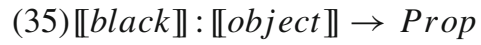

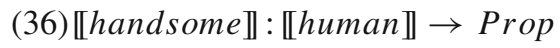

\footnotetext{
11 Ranta (1994) did not consider different classes of adjectives and we think that he mainly had intersective adjectives in mind when considering this.

12 It may be worth remarking that the notion of 'subset' (Nordström et al. 1990) as discussed in Section 3.3 of Ranta (1994) has been abandoned since it cannot be properly incorporated into MTTs such as MartinLöf's type theory.
} 
A modified $\mathrm{CN}$ like handsome man is interpreted as the following $\Sigma$-type:

(37) $\Sigma m: \llbracket m a n \rrbracket . \llbracket h a n d s o m e \rrbracket(m)$

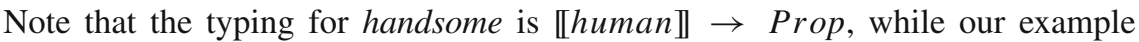
involves the type man. The reason this example is well-formed is due to the subtyping relation $\llbracket m a n \rrbracket<\llbracket h u m a n \rrbracket$ and the fact that subtyping relations propagate through the type constructors (in our example, $\Sigma$, Luo 2010)

(38) $\Sigma m: \llbracket m a n \rrbracket . \llbracket h a n d s o m e \rrbracket(m)<\Sigma m: \llbracket h u m a n \rrbracket . \llbracket h a n d s o m e \rrbracket(m)$

Let us see what this account predicts as regards inference. Intersective adjectives are associated with two types of inference. The first one is shown below (we show this both in informal notation as well as using the MTT semantics notation and given that CNs are taken to be types):

(39) From $\operatorname{Adj}(\mathrm{N})(\mathrm{x})$ infer $\mathrm{N}(\mathrm{x})$.

(40)From $\Sigma x: \llbracket N \rrbracket . \llbracket A d j \rrbracket(x)$ infer $x: \llbracket N \rrbracket$.

This inference is very easily taken care of, given that the first projection of the $\Sigma$ is always a coercion, in effect for any $\Sigma$ type of the form $\Sigma(A, B)$, we can always infer $A$. Subtyping does the job here. The second inference associated with intersective adjectives is a little bit trickier theoretically. In the mainstream Montagovian literature on adjectives, e.g. Kamp (1975); Partee (2007), the inference schema for intersective adjectives is as follows:

(41) From $\operatorname{Adj}(\mathrm{N})(\mathrm{x})$ infer $\mathrm{N}(\mathrm{x}) \wedge \operatorname{Adj}(\mathrm{x})$.

(42)From $\Sigma x: \llbracket N \rrbracket . \llbracket A d j \rrbracket(x)$ infer $x: \llbracket N \rrbracket \wedge \llbracket A d j \rrbracket(x)$.

But what does $\operatorname{Adj}(\mathrm{x})$ mean? It means that $A d j$ is true of $x$. In the case of black man it means that $x$ is a man and $x$ is black. But what does it mean in terms inference? Well, what it means is that if we have $\operatorname{Adj}\left(N_{1}\right)(x)$ and given a noun $N_{2}$, where $N_{1}<N_{2}$, then it should follow that:

(43)From $\operatorname{Adj}\left(\mathrm{N}_{1}\right)(\mathrm{x})$ infer $\mathrm{N}_{1}(\mathrm{x}) \wedge \operatorname{Adj}\left(\mathrm{N}_{2}\right)(\mathrm{x})$.

In practical terms and taking black as our example, this means that for every $A$ and $B$ where $A<B$, we have black $A<$ black $B$. In case no relation between $A$ and $B$ exists or if the subtyping relation is reversed, no inference should be possible. Indeed, given the subtyping relations and the fact that subtyping relations propagate through the constructor types, we predict the desired inferences.

Intersective adjectives can be interpreted in this respect by a simple predicate type, declaring the subtyping relations and interpreting adjectival modification as a $\Sigma$ type. However, this will overgenerate for subsective adjectives. This is because in this case we have to find a way of deriving the first inference associated with intersective 
adjectives but not the second, thus we need to take care of the following inference only:

(44) $\operatorname{Adj}(\mathrm{N}) \Rightarrow \mathrm{N}$.

Thus, in the case of an adjective like small we need to predict that from small $N$, $N$ follows but given $A$, where $N<A$, small $A$ does not follow. To give an example, one should not be able to deduce small animal from small elephant. The solution here is to use universes in the typing for subsective adjectives, in effect having the type for subsective adjectives ranging over the universe $\mathrm{CN}$. Thus, an adjective like small will receive the following type:

(45) $\Pi$ A: CN. $(A \rightarrow$ Prop $)$

The above idea has been proposed by Chatzikyriakidis and Luo (2013) and it is basically an implementation of the intuition that subsective adjectives are only relevant for the particular $\mathrm{CN}$ they modify in each case. Thus, a small elephant is only small with respect to elephants, a skilful surgeon is only skilful for a surgeon, and so on. Using the type proposed in (45), we can have different instances of a subsective adjective, say $P$, depending on the choice of $A$, with $A: \mathrm{CN}$. This account of intersective and non-subsective adjectives relies on the following assumptions: (a) CNs are types, (b) adjectives are predicates (or lower function types, see discussion in Sect. 3.2.2), (c) predicates may be polymorphic. The welcome result in this approach is that inferential properties are derived via typing only and no extra axioms in the form of meaning postulates are needed.

\subsection{Non-subsective Adjectives}

The next class of adjectives is classified into two further subclasses, that of privative and that of non-committal adjectives. Privative adjectives are those adjectives that give rise to the following inferential schema:

(46)From $\operatorname{Adj}(\mathrm{N})$ infer $\neg \mathrm{N}$.

(47)From $\Sigma x: \llbracket N \rrbracket \cdot \llbracket A d j \rrbracket(x)$ infer $x: \llbracket N \rrbracket \rightarrow \perp$.

Privative adjectives include adjectives like fake and former. The privative class is potentially the most problematic class, since there is no general consensus that these adjectives do indeed give rise to such inferences. For example, for the case of fake, Partee (2007) has argued (convincingly in our opinion) that adjectives like fake are not really privative. Partee argues that privative adjectives are in fact interpreted as subsective (Partee 2007, 2010). The idea is that in cases of privative modification the interpretation of the $\mathrm{CN}$ is coerced to include the denotations of CNs modified by 
privative adjectives. Thus, in the case of (48) and (49), Partee argues that the denotation of fur is expanded to include both real and fake furs:

(48) I don't care whether that fur is fake fur or real fur.

(49)I don't care whether that fur is fake or real.

Thus, in fake fur, fur is coerced to include fake furs as well.

A potentially similar idea was first considered, independently with Partee (2007, 2010), by the second author in Luo (2011a) where he proposed to use disjoint union types to represent adjectival modifications by privative adjectives, and was further studied by us in Chatzikyriakidis and Luo (2013). The idea is as follows: We define $G_{R}$ and $G_{F}$ to be the type of (real) guns and fake guns, respectively. Then,

$$
G=G_{R}+G_{F}
$$

represents the type of all guns. It consists of objects of the form $\operatorname{inl}(r)$ and $\operatorname{inr}(f)$, where $r: G_{R}$ and $f: G_{F}$. The associated injection operators $i n l: G_{F} \rightarrow G$ are declared and $i n r: G_{R} \rightarrow G$ as coercions:

$$
G_{R}<_{i n l} G \text { and } G_{F}<_{i n r} G
$$

The following predicates can now be defined: real_gun and fake_gun of type $G \rightarrow$ Prop:

$$
\begin{gathered}
\text { real_gun }(\operatorname{inl}(r))=\text { True and real_gun }(\operatorname{inr}(f))=\text { False; } \\
\text { fake_gun }(\operatorname{inl}(r))=\text { False and fake_gun }(\operatorname{inr}(f))=\text { True. }
\end{gathered}
$$

It is easy to see that, for any $g: G$,

(50) real_gun $(g)$ iff $\neg f a k e \_g u n(g)$.

The following interpretations can be now given (both are true): for $g: G_{R}$ :

(51) $\llbracket g$ is a real gun $=$ real_gun $(g)$

and for $f: G_{F}$,

(52) $\llbracket f$ is not a real gun $=\neg r e a l \_g u n(f)$

Given the above, it is not difficult to see that the sentences like those below can easily be interpreted as expected:

(53)Is that gun real or fake?

(54) A fake gun is not a gun. 
Note that in the second example gun is taken to mean real gun. This needs some explanation. According to the Partee explanation, without the coercion of gun to include fake guns, the adjective real would also be redundant (since all guns would be real guns). So, in the above example we take this to mean that a fake gun is not a real gun.

Adjectives like former have similar problems, given that there is no consensus on the inferences they give rise to. Some people accept the judgments associated with privative adjectives while others categorize them as non-committal, i.e. as giving rise to no inference whatsoever The two options are given below:

(55)Former(N) $\Rightarrow \neg \mathrm{N}$.

(56) Former(N) $\Rightarrow$ ?.

On the assumption that former is a privative adjective, one can pursue an analysis similar to the one given for fake. However, it seems to us that in giving a correct account of former, one needs to take into consideration the time parameter associated with former and that the correct inference associated with former should be that from former $N$ one can infer $N$ in some past time. One might argue that this should also involve the inference that former $N$ implies the negation of current $N$ but this seems to be disputed by data like the following:

(57)The former president, which happens to be the current one as well, addressed the crowd

The relevant intuition here seems to be that former $N$ implies $\neg$ current $N$, i.e. that $\neg N$ holds at the current time, but only if there is a time in between the current time and the past time where former $N$ was true, where $\neg N$ was the case. Thus, a former president that was re-elected after having a break from presidency can also be the current president. But, it seems that a re-elected president without any break from presidency cannot be considered a former president, but a current president. If this is true, analyses in the style of Dowty (1981), i.e. a predicate modifier approach, where the noun does not hold of its argument in the evaluation time but a time preceding it overgenerate. We present a more recent version of this idea presented by Morzycki (2014) where worlds are substituted with Kratzerian situations (Kratzer 1989):

$$
\begin{aligned}
& \underset{\text { prormer president } \rrbracket}{ } \underset{\text { president }(x)(s)]}{ }=\lambda x \cdot \lambda s \cdot \exists s^{\prime}\left[s^{\prime}<s \wedge \operatorname{president}(x)\left(s^{\prime}\right) \wedge\right. \\
&
\end{aligned}
$$

We want to propose a refinement of this idea in MTTs. The idea is to use CNs indexed with a time parameter. Following Chatzikyriakidis and Luo (2014b), we introduce a type Time of times to deal with the parameter to time (e.g. Ranta 1994 also uses a similar type to deal with tense). Over Time, we have a precedence relation $\leq$ as well as

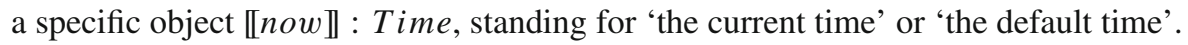
The $\leq$ relation conforms to the usual properties, irreflexivity, transitivity, asymmetry, 
connectedness and density. Time can be also specified as an inductive type, in the sense proposed in Chatzikyriakidis and Luo (2014b). This proposal is shown below:

(59)【date $\rrbracket: D A T E \rightarrow$ Time.

where $D A T E$ consists of the triples $(y, m, d)$ where $y$ ranges over integers to represent years, $m$ over Jan to Dec to represent months, and $d$ over the days $1,2, \ldots$ to represent days.

With these assumptions in place, we can further assume that CNs are indexed with a time parameter. Thus, a common noun like mayor is not interpreted as a plain $\mathrm{CN}$ but as a family of types indexed by the parameter $t$ :Time:

(60)mayor $(t): \mathrm{CN}$.

(61) $\llbracket$ former mayor $\rrbracket=\neg$ mayor $($ now $) \wedge \exists t:$ Time. $t<$ now $\wedge$ mayor $(t) \wedge$ $\exists t_{1}:$ Time, $t_{1} \neq t \wedge t_{1} \leq$ now $\wedge \neg$ mayor $\left(t_{1}\right)$.

Former is defined in this respect as $\llbracket$ former $\rrbracket:($ Time $\rightarrow \mathrm{CN}) \rightarrow \mathrm{CN}$, obtained by abstracting mayor in (61):

(62) $\llbracket$ former $\rrbracket(p)=\neg p($ now $) \wedge \exists t$ : Time. $t<$ now $\wedge p(t) \wedge \exists t_{1}:$ Time, $t_{1} \neq$ $t \wedge t_{1} \leq$ now $\wedge \neg p\left(t_{1}\right)$.

Similar ideas can be developed for other temporal adjectives like past, current $^{13}$ Another thing we should look at is the typing. The typing associated with former as we have said is $\llbracket$ former $\rrbracket:($ Time $\rightarrow \mathrm{CN}) \rightarrow \mathrm{CN}$. It involves a time parameter and also returns a $\mathrm{CN}$ instead of a proposition. The latter will predict that adjectives like former cannot be used predicatively. ${ }^{14}$ However, giving this type will mean that any $\mathrm{CN}$ type can be combined with former, a fact that is not true. For example, in general non-animate CNs are not possible with former:

\section{(63)\# Former table/house/piano/man/human}

Furthermore, cases of nouns that denote a permanent property, in effect individual level nouns, are also not good with former:

\footnotetext{
13 It has to be noted however that in order to do full justice to this kind of adjectives one has to engage into the issue of temporal sensitivity of nouns. In our case, this means that we have to look at the way the temporal index of CNs interacts with the rest of the sentence. Unfortunately, such engagement cannot be done in this paper for obvious reasons of space. This however as well as the more general issue of providing a solid temporal theory using MTTs is one of the things that we are currently looking at. For more information on the temporal sensitivity of CNs, the interested reader is redirected to Enc (1981), Musan (1995), Tonhauser (2002) for thorough discussions and proposals on temporal sensitivity.

14 Please see the remark on predicativity at the end of this section.
} 


$$
\overline{\text { mayor, student...: } \mathrm{CN}_{s}} \frac{A: \mathrm{CN}_{s}}{A: \mathrm{CN}}
$$

Fig. 2 Some introduction rules for $\mathrm{CN}_{S}$

(64)\# Former man/human

From these data, it seems that former combines with stage level nouns that are also

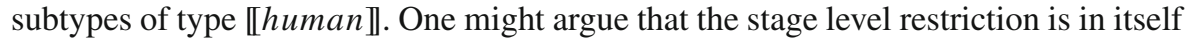
not strange, given that the semantics of former specify a change from a time where an $x$ is true of the property and a time where $x$ is not true any more. For CNs that denote a permanent property, e.g man or human, combination with former is thus incompatible because such change is inherently contradictory. It is worth noting, in support of the argument we are making here, that cases where former actually combines with individual level nouns, these are coerced into receiving a stage level interpretation:

\section{(65)Her former man}

In the above example man basically has the meaning of boyfriend, husband, in effect it is turned into a stage level noun. The way this kind of coercions precisely work is a matter well beyond the scope of this paper. However, coercive subtyping is a mechanism that has been argued to be fit for dealing with a wide range of linguistic coercions, see e.g. Luo (2011b), Asher and Luo (2012). What is relevant here, is that former needs a stage level noun to combine with and in order to combine with individual level nouns, a some kind of coercion into stage level has to be performed.

In order to express this idea, we introduce the subuniverse $\mathrm{CN}_{S}$ such that $\mathrm{CN}_{s}<\mathrm{CN}$ that contains the (names) of the types for stage level CNs. We follow pretty much the same idea as in the construction of the $\mathrm{CN}$ universe, namely the fact that $\mathrm{CN}_{S}$ should be open. The most important rule is one where types in $\mathrm{CN}_{s}$ would also be in CN. In Fig. 2, we show two of these rules, one introducing stage level nouns into the $\mathrm{CN}_{s}$ universe and one denoting that every name $\mathrm{CN}_{s}$ is also in $\mathrm{CN}$.

In general, one can argue that the category of privative adjectives can be suspended. The various adjectives of this class can be in fact argued to be either subsective or non-committal rather than privative. With this remark, we can now look at the category of not committal adjectives. This category involves adjectives that do not give rise to any inference whatsoever:

(66) $\operatorname{Adj}(\mathrm{N}) \Rightarrow ?$

Classical cases of this category include modal adjectives like alleged or possible. Let us look at the adjective alleged. What does alleged $N$ mean? What is an alleged murderer for example? An alleged murderer is someone who has been alleged at least by someone that he is a murderer. The assumption we are going to make is that an alleged murderer is a murderer in someone's belief context. Then, we can formulate belief contexts in the sense of Ranta (1994): a belief context is a sequence 
of assumptions that an agent $p$ has made. More precisely, the belief context of an agent $p, \Gamma_{p}$, is a context of the following form:

$$
\Gamma=x_{1}: A_{1}, x_{2}: A_{2}\left(x_{1}\right), \ldots, x_{n}: A_{n}\left(x_{1}, \ldots, x_{n-1}\right)
$$

Based on this, Ranta proposes the belief operator $B_{p}$, defined as

$$
B_{p} A=\Pi \Gamma_{p} . A=\Pi x_{1}: A_{1} \ldots \Pi x_{n}: A_{n}\left(x_{1}, \ldots, x_{n-1}\right) . A .
$$

As a consequence, $B_{p} A$ is true if and only if $A$ is true in $\Gamma_{p}$. Now, given the above, we can interpret alleged as follows for $A_{N}$ : CN being the interpretation of a common noun $N$ :

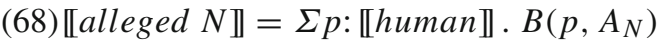

where $B(p, A)=\Pi \Gamma_{p} . A$ with $\Gamma_{p}$ being the belief context of $p: \llbracket$ human $\rrbracket .{ }^{15}$

Again, as in the case of former, one should restrict the scope of alleged. This is because the definition in (69) will overgenerate, since it will predict combinations like alleged chair and alleged democracy to be possible. It seems that alleged, similarly to former, can only combine with stage level CNs. In this sense, we update the definition for $\llbracket A_{N} \rrbracket: \mathrm{CN}_{s}$.

\subsubsection{A Note on Unknown Beliefs}

The definition proposed for alleged involves existential quantification over an agent $p$ : «human $\rrbracket$ and then a judgment concerning the belief context of this agent. The problem that arises here is the issue of unknown beliefs, simply put how we define belief contexts for unknown agents. There are a number of ways to deal/argue with this problem. The first would be to assume that we know beforehand the belief contexts of

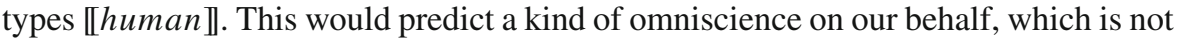
very plausible. Another way at looking at the matter, would be to define alleged or any other word involving belief contexts and unknown agents as involving quantification

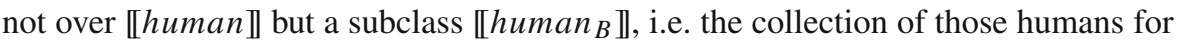
which their beliefs are known $\left(\llbracket\right.$ human $_{B} \rrbracket<\llbracket$ human $\left.\rrbracket\right)$. Lastly, there is another way, which to our opinion might give a good solution to this problem but its formal details cannot be really specified here. The idea is as follows: when we talk about other people's beliefs what we are actually representing is our perspective of the other person's belief context, i.e. our representation of this context depending on the information we have. Sometimes this representation might exactly match the other person's belief context, sometimes it can be a close approximation and sometimes it might be even totally different. So when someone utters 'John believes that he opened

\footnotetext{
15 This is the analog of a formula that involves existential quantifications. One may turn such types into propositions by means of the following operation: for any type $A, \operatorname{Exists}(A)=\exists x$ :A.True. Then, with this mechanism, (69) can be represented as the proposition $\exists p$ : $\llbracket$ human $\rrbracket . \operatorname{Exists}\left(B\left(p, A_{N}\right)\right)$.
} 
the door', what we really get is not that the proposition opened(the_door $)($ John $)$ is part of $\Gamma_{j}$ but rather part of $\Gamma_{j(u)}$, that is John's belief context as seen by the utterer $u$. This is we believe an interesting idea that would be able to distinguish and predict different contexts in cases like the following:

(69) A: John believes that Mary is a left winged radical. B: No, John does not believe this.

In the above cases, we have two distinct utterers with different versions of John's belief context, $G_{j(u 1)}$ and $G_{j(u 2)}$. These cases would be impossible to get by assuming one $G_{j}$ context. In this sense, the belief context is relativized to the utterer in each case. Thus, in cases of unknown agents, this b elief context might just be the minimal context including $B\left(p, A_{N}\right)$ in the case of alleged and nothing more. We take this to be a promising way to deal with these cases. However, we have to look at the formal details behind such a proposal. This task cannot be taken up in this paper. Similar considerations apply to the other cases where belief contexts are used in this paper.

\subsubsection{A Note on Predicativity}

It is a well-known fact that a number of adjectives cannot be used predicatively but only attributively. We have considered adjectives of this sort so far, e.g. alleged or former are of this type. An explanation for the behaviour of this class of adjectives comes from Coppock (2008) who argues that adjectives that cannot be used predicatively are not semantically predicative. In standard terms, this means that the adjective is not a predicate. It has been pointed out to us that if we assume a lower predicate type for adjectives, problems arise w.r.t non-predicative adjectives. This is correct. However, we do not assume that all adjectives are predicates. We do assume that there are no higher order types associated with adjectives, but we do assume that adjectives might not be predicates. We have already exemplified the latter point for adjectives like former where the typing for former was (Time $\left.\rightarrow \mathrm{CN}_{S}\right) \rightarrow \mathrm{CN}_{s}$. In effect, for non-predicative adjectives the idea is that these are function types that return a $\mathrm{CN}$ type rather than a predicate (i.e. returning an element of type Prop). Similar considerations apply to the adjective alleged and generalizing, to all non-predicative adjectives.

\section{Gradable Adjectives and Multidimensional Adjectives}

\subsection{Gradable Adjectives}

Adjectives like small and large do not only belong to the category of subsective adjectives but have a further distinguishing property, i.e. they are also gradable adjectives. In general, by gradable adjectives we mean the class of adjectives that involve some kind of grading parameter that allows them to be quantified according to it. For example, in the case of small and large the grading parameter is size. Gradable adjectives are traditionally found in comparative or superlative forms and can be further modified by grading adverbs. The usual assumption made in the literature on gradable adjectives, as already noted in the inroduction and which conforms to our intuitions about this 
type of adjectives, is that some kind of measurement is involved. Then, two approaches are found depending on whether this parameter is formally encoded in the definition for gradable adjectives or not. The type of a gradable adjective differs minimally from the type of a non-gradable one. Simply put, gradable adjectives have a degree argument, while non-gradable ones don't. For example, on the assumption that small and large are given lower predicate types, (i.e. $e \rightarrow t$ ), then the modified typing to further deal with gradability will be $d \rightarrow(e \rightarrow t)$. Example (70), showing the definition for adjective small under this view is repeated below:

(70) small $\rrbracket=\lambda d:$ Degree. $\lambda x:$ e.Height $(x) \leq d$

Proponents of such an approach can be found in Bartsch and Vennemann (1973), Von Stechow (1984), Heim (2000) among others. The other option for treating gradable adjectives is to assume that they involve the same typing as non-gradable ones. The difference between the two is that gradable adjectives, even though being predicates from individuals to truth values, they further involve partially ordered domains. Gradable adjectives impose a partitioning of this partially ordered domains. For objects $x$ that fall into the upper side of the domain imposed by adjective $A, A(x)$ is true while for objects $y$ on the lower side of the scale, $A(y)$ is false. This is the approach that Kennedy (1999) calls the Vague Predicate Approach. Proponents of such an approach can be found in Lewis (1970), McConnell-Ginet (1973), Klein (1980), van Benthem (2012). The list of accounts for gradable adjectives is quite long to be fully mentioned and the interested reader is redirected to Kennedy (1999) for more information on these accounts and additional references. Another nice and most recent overview of the two approaches is Lassiter (2014).

In what follows, we are going to propose a way of dealing with gradable adjectives in MTTs. The account is based on earlier treatments proposed by the authors as regards comparative adjectives (Chatzikyriakidis and Luo 2014b) and it is in line with the approach that gradability involves an implicit grade parameter but transferred to an MTT setting. The account uses indexed types, in particular CNs that are indexed by a degree parameter. Let's consider shorter than as an example, taking heights to be measured by the type «Height $\rrbracket$ of numbers such as $1.70 .{ }^{16}$ We are then led to consider

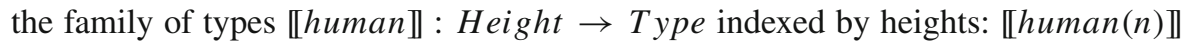
is the type of humans of height $n$. Then, shorter than is defined as follows: ${ }^{17,18}$

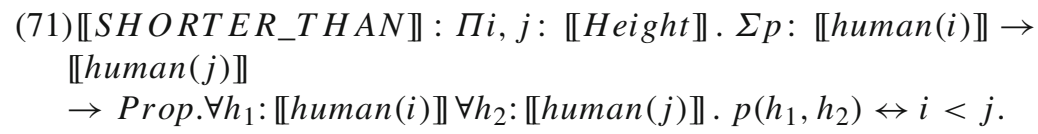

\footnotetext{
16 Here we do not spell out the type «Height . One might take Height to be the type of natural numbers and use 170 to stand for 1.70 , etc.

17 The transitive properties of comparatives are not encoded in this example for reasons of simplicity. One may very well do so having as a guide the previous entry without measures.

18 The definition involves a bi-implication, given that if the height of human $x$ is less than the height of another human $y$, then it is also the case that $x$ is shorter than $y$. The definition also works as an implication.
} 
(72) 【shorter than $\rrbracket(i, j)=\pi_{1}($ SHORTER_THAN $(i, j)): \llbracket \operatorname{human}(i) \rrbracket \rightarrow$ $\llbracket \operatorname{human}(j) \rrbracket \rightarrow \operatorname{Prop}$

With this account in line, one can propose that positives are just special instances of comparatives. The only difference in this case is that $<$ is between a degree provided by the [human(n)》 argument and a contextually provided degree parameter. The typing in this respect is different than comparatives (missing one indexed argument), being as follows: ${ }^{19}$

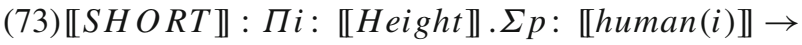

Prop. $\forall h_{1}: \llbracket h u m a n(i) \rrbracket \cdot p\left(h_{1}\right)$

$\leftrightarrow i<n$.

(74) $\llbracket \operatorname{short} \rrbracket(i)=\pi_{1}(\operatorname{SHORT}(i)): \llbracket \operatorname{human}(i) \rrbracket \rightarrow$ Prop

A question that naturally comes to mind and has been the subject of pretty much all degree based accounts that assume a standard contextual degree parameter, is where do we get this parameter from. The idea is that the context (in the MTT sense) that the adjective is evaluated at, provides this value. More specifically, a polymorphic function STND (standing for 'standard') is assumed that takes a gradable adjective as an argument and returns the contextual parameter relevant for each adjective: ${ }^{20}$

(75) $\llbracket S T N D \rrbracket=\lambda A: C N . \lambda i: D . \lambda P: A_{i} \rightarrow \operatorname{Prop} . \exists n_{1}: N a t . n_{1}=n \wedge i<>n$

Now, the exact value of $n$ (or a range of values) might be specified in the context. But sometimes it might not. For example assume that we hear out of the blue that George is tall. One way is to interpret this as matching the value that our knowledge context has or to assume that there is a value that we do not know yet in the context of the conversation (here we take this to be $\Gamma$ ): ${ }^{21}$

(76) $K_{s p}=\llbracket(S T N D($ Height $)(i)($ tall $) \rrbracket=(180=n) \wedge i>n)$

(77) $\Gamma=\llbracket\left(S T N D(\right.$ Height $)(i)($ tall $\left.) \rrbracket=\left(\exists n_{1}: N a t . n_{1}=n\right) \wedge i>n\right)$

Note that the value in $\Gamma$ might be elaborated via context extension. Following Ranta (1994) particular, we define a mapping $f: \Delta \rightarrow \Gamma$, where everything that is in $\Gamma$ is also in $\Delta$ plus some potentially extra information. ${ }^{22}$ Also, this idea of using TT contexts will be natural in cases the standard value is way-off the one usually found in

\footnotetext{
19 Where $n$ : «Height $\rrbracket$, the contextual degree parameter.

$20<>$ stands for either $<$ or $>$. For type $D$ see the following discussion.

$21 K_{s p}$ stands for the speaker's knowledge context.

22 This corresponds to what Boldini (2000) called logical inference between contexts. In particular $\exists n_{1}: N a t . n_{1}=n$ will be reduced to $180=n$ by the $\exists$ elimination rule.
} 
the default context. Consider for example the context of all statements pertaining to basketball. In there, and given the nature of the sport, the meaning of tall and short is inevitably different. ${ }^{23}$

Lastly, in order to deal with different types of degrees on a more general scale, we can introduce the universe of all degree types, $D$. All the types in this universe are totally ordered and dense, i.e. they respect the following axioms:

(78) $T R=\Pi$ A:D. $\forall d, d_{1}, d_{2}: A . d \leq d_{1} \wedge d_{1} \leq d_{2} \rightarrow d \leq d_{2}$

$A N T$ I SY M = П A:D. $\forall d, d_{1}: A . d \leq d_{1} \wedge d_{1} \leq d \rightarrow d \leq=d_{1}$

$R E F L=\Pi A: D . \forall d: A . d \leq d$

$D E N S=\Pi A: D \cdot \forall d, d_{1}: A \cdot d \leq d_{1} \rightarrow \exists d_{2}: A, d \leq d_{2} \leq d_{1}$

In the universe $D$, one can find types $\llbracket H e i g h t \rrbracket, \llbracket W e i g h t \rrbracket, \llbracket W i d t h \rrbracket: D$ among other types.

\subsection{Multidimensional Adjectives}

Multidimensional adjectives are those adjectives that can be quantified across different dimensions. Such cases include adjectives like sick, healthy etc. Two different classes of multidimensional adjectives are distinguished (Sassoon 2012), positive and negative. Basically, every positive adjective has a negative counterpart, its antonym. Thus, for the positive adjective healthy, we get the negative sick. The difference is the form of quantification over dimensions in each case. Positive adjectives involve universal quantification over dimensions, while negative adjectives existential quantification. Thus, for someone to be considered healthy, s/he must be healthy in all dimensions, whereas sick, it suffices to be sick across one dimension only. In order to see this, one can use the exception phrase except. This phrase is only compatible with universal quantification. As can be seen below, only healthy is compatible with except, sick being infelicitous:

(79)Dan is healthy except with respect to blood pressure

(80)\# Dan is sick except with respect to blood pressure

This idea put forth by Sassoon can be implemented in an MTT setting using an inductive type for multiple dimensions. Let us explain. Consider an adjective like healthy. In order for someone to be considered healthy, one must be able to universally quantify over a number of 'health' dimensions such as cholesterol, blood pressure etc. To formalize this, we can introduce the inductive type Health of type $D$ as follows: ${ }^{24}$

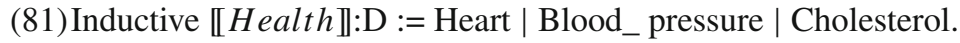

\footnotetext{
23 Another consequence of this approach is that given the polymorphic type of the function, the $n$ is always relativized to both $A: C N$ and $i: D$. c.)Thus, for a polymorphic adjective like small, the contexrualized value

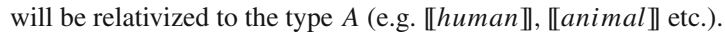

24 The inductive type Health in $\mathrm{CN}$ is the finite type (also called an enumeration type), sometimes written as $\{$ Heart, Blood_pressure, Cholesterol $\}$.
} 
We now define the adjective Healthy to be of the following type, where we use

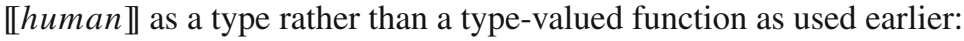

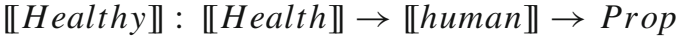

We can use this as a primitive to define healthy and sick as follows:

(83) $\llbracket$ healthy $=\lambda x: \llbracket h u m a n \rrbracket . \forall h: \llbracket$ Health $\rrbracket . H e a l t h y(h)(x)$

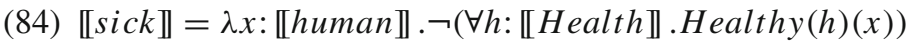

The idea here is to $[$ Health $\rrbracket$ as an inductive type, in order to encode all the different dimensions we need. This is one way of dealing with multidimensional adjectives in MTTs. Of course, there are a number of issues in case one wants to further give a full theory of gradable and multidimensional adjectives. Our goal was to show an initial way of approaching these kind of adjectives in MTTs. The interested reader who wants to further investigate the issue of gradability and multidimensionality of adjectives is directed to Lassiter (2014) and references therein for gradability and Sassoon (2012) for multidimensional adjectives.

\section{Adverbial Modification}

The literature on adverbs in MTTs is rather poor. The only paper specifically dealing with adverbial modification is Chatzikyriakidis (2014). ${ }^{25}$ However, adverbs have been also treated in Chatzikyriakidis and Luo (2014b) as part of a discussion on Natural Language Inference (NLI). There, a first approach of some aspects of adverbial modification like veridicality, non-veridicality, adverbial typing and intensional adverbs among others has been attempted. Here, we extend the approach of Luo (2011a), Chatzikyriakidis (2014), Chatzikyriakidis and Luo (2014b) to further adverbial classes and deepen the analysis given there.

The first thing to discuss is the notion of typing, given that MTTs are many sorted and in general are quite different typed systems than the ones presented in different versions of simple type theory. The proposals put forth so far in the literature are based on the second author's proposal (Luo 2011a,b), subsequently followed in Chatzikyriakidis and Luo (2014b), Chatzikyriakidis (2014), according to which, VP adverbs receive a polymorphic type extending over the universe $\mathrm{CN}(85)$, while sentence level adverbs are just functions from propositions to propositions (86):

(85) $\Pi$ A: CN. $(A \rightarrow$ Prop $) \rightarrow(A \rightarrow$ Prop $)$

(86) Prop $\rightarrow$ Prop

25 One of the reasons for this is that researchers found it difficult to give adverbial typings when CNs are interpreted as types. The first to discuss adverbial typings was Luo (2011a) who proposed to use the universe $\mathrm{CN}$ and polymorphism to solve this problem. This proposal was followed in Chatzikyriakidis (2014) and also in this paper. 


\subsection{Veridicality}

A very basic distinction in terms of the semantic properties of adverbs, in particular the inference patterns that they give rise to, concerns what has been dubbed as veridicality. Veridicality is found in both VP and sentence level adverb. Veridicality in the case of sentence adverbs means that $A d v(P)$ presupposes $P$ whereas in the case of VP adverbs $V(P(x))$ presupposes $P(x)){ }^{26}$

In order to take care of veridical adverbs Chatzikyriakidis and Luo (2014b), a technique similar to the one used for comparatives is proposed in Chatzikyriakidis and Luo (2014b). First, an auxiliary object veridical is defined (two definitions one for VP and one for sentence adverbs, $V E R_{\text {Prop }}$ and $V E R_{V P}$ respectively), and then veridical adverbs are defined as the first projection of this auxiliary object ( $A D V_{v e r-P r o p}$ and $\left.A D V_{\text {ver }-V P}\right)$ :

(87) $\llbracket V E R_{\text {Prop }} \rrbracket: \Pi v:$ Prop. $\Sigma$ p: Prop.p $\supset v$

(88) $\llbracket V E R_{V P} \rrbracket: \Pi A: \mathrm{CN} . \Pi v: A \rightarrow$ Prop. $\Sigma p: A \rightarrow$ Prop. $\forall x: A . p(x) \supset v(x)$

(89) $\llbracket A D V_{\text {ver-Prop }} \rrbracket=\lambda v: \operatorname{Prop} . \pi_{1}\left(V E R_{\text {Prop }}(v)\right)$

(90) $\llbracket A D V_{v e r-V P} \rrbracket=\lambda A: \mathrm{CN} . \lambda v: A \rightarrow \operatorname{Prop} . \pi_{1}\left(V E R_{V P}(A, v)\right)$

Let us see how this approach works in more detail. We will exemplify this with the sentence adverb case (the reader can then work out the VP-adverb case). According to the above, a sentence adverb like fortunately will be defined as in (92), i.e. as the first projection $\pi_{1}$ of (87):

(91) [Fortunately $\rrbracket: \Pi v:$ Prop. $\Sigma$ p:Prop. $p \Rightarrow v$

(92) $\llbracket$ fortunately $=\lambda v: \operatorname{Prop} . \pi_{1}$ (FORTUNATEly $\left.(v)\right)$

The type of (92) is Prop $\rightarrow$ Prop: it takes an argument $v:$ Prop and returns the first component of the pair FORTUNATELy $(v)$, which is also of type Prop. Now, let us consider the following inference:

(93) Fortunately, John went $\Longrightarrow$ John went

To see that (93) is the case, we only need to realise that the second component of FORTUNATELY $(v)$ is a proof of

(94) $\llbracket$ fortunately $\rrbracket(v) \Rightarrow v$

Taking $v$ to be $\llbracket J o h n$ went $\rrbracket,(94)$ is the semantic representation of (93).

Note that what we have presented here only deals with the veridical property and does not say anything further about the semantics of the adverbs in each case. In order to get into the specifics of each veridical adverb, more information will be introduced,

${ }^{26}$ With $P: A \rightarrow$ Prop, $x: A$ and $A: \mathrm{CN}$ 
potentially in the form of a conjunction, but this is something that we have not looked at yet.

\subsection{Intensional Adverbs}

Veridicality/non-veridicality, as already mentioned, is just one of the aspects associated with the meaning of adverbs. This is however not sufficient to deal with a number of other aspects of the semantics of adverbials. For example, cases of adverbs like intentionally or epistemic adverbs like possibly or allegedly cannot be treated in this manner. One of the reasons for this is that this type of adverbs creates what we call opaque contexts. The latter type of adverbs, i.e. epistemic adverbs, creates opaque contexts for both the subject and the object, while the former, i.e intentionally, only for the object:

(95) Oedipus allegedly married Jocaste.

(96) Oedipus intentionally married Jocaste.

From (95), we have:

(97) Oedipus allegedly married Jocaste $\nRightarrow$ the son of Laius allegedly married Jocaste (98)Oedipus allegedly married Jocaste $\nRightarrow$ Oedipus allegedly married his mother

On the other hand, from (96) we have:

(99) Oedipus intentionally married Jocaste $\Rightarrow$ The son of Laius intentionally married Jocaste

(100) Oedipus intentionally married Jocaste $\nRightarrow$ Oedipus intentionally married his mother

In order to deal with these data, the first author Chatzikyriakidis (2014) uses the type theoretic notion of context similarly to the way used by Ranta (1994) and also here in this paper.

The meaning of intentionally is taken to be the following: there exists an agent $p$, that did something $x$ intentionally, i.e. agent $p$ believes that he did $x$. This analysis faces a number of problems. It is certainly true that intentions and beliefs are related in a number of ways but it seems that approaching intentionally via belief contexts might lead us to problems. The most obvious problem, concerns cases like the following:

(101) I accidentally bumped into someone and I believe that I did it

One would expect this sentence not to be correct on the assumption that accidentally is a antonym of intentionally.

A better analysis of the meaning of intentionally would be something like the following: $A$ intentionally $P$ means that $A$ has the intention $P$ and furthermore fulfilled 
this intention, i.e. $P$ holds. In order to formalize this, we introduce the notion of intention contexts, which represent an agent's collection of intentions.

We can represent $p$ 's intentional context as a number of judgements $x: A$ (A: Prop) corresponding to intentions, this agent holds: ${ }^{27}$

$$
\mathcal{D}_{p}=x_{1}: A_{1}, \ldots, x_{n}: A_{n}\left(x_{1}, \ldots, x_{n-1}\right)
$$

From this, and again following (Ranta 1994), we can construct a generalized intention operator by binding all the variables in $\mathcal{D}_{p}$

(103) $I_{p} A=\Pi \mathcal{D}_{p} . A=\Pi x_{1}: A_{1} \ldots \Pi x_{n}: A_{n}\left(x_{1}, \ldots, x_{n-1}\right) . A$

With these ideas in mind, one can put forward an account of intentionally as follows:

$(104) \llbracket$ Intentionally $\rrbracket=\lambda x: \llbracket h u m a n \rrbracket \cdot \lambda P: \llbracket h u m a n \rrbracket \rightarrow \operatorname{Prop} . I_{x}(P(x)) \wedge$ $\Gamma(P(x))$

Thus, in the case of Oedipus intentionally married Jocaste, we get a paraphrase that Oedipus had the intention of marrying Jocaste and he did so. In (99), we see that the $x$ is bound. If we assume $E q($ Person, $O, S o L)$ in the global context, then substituting $O$ for $x$ and then $S o L$ for $O$, we get the following( $M$ stands for married and $J$ for Jocaste):

(105) 【Intentionally $O(M(J)) \rrbracket=I_{S o L}(M(J(S o L)) \wedge \Gamma(M(J)(S o L))$

Thus, (99) is predicted. On the other hand, in order to prove that Oedipus intentionally married his mother, we need to have $M(O, M o O)$ in the intention context of Oedipus. If we assume that the intention context of Oedipus is known and according to the standard reading of the story does not involve the aforementioned intention, then this does not follow. If we assume that Oedipus' intention context is unknown, we cannot prove it nor disprove before this information becomes available.

For cases of opaque to both the subject and the object adverbs, one needs of course a different analysis. For example, for allegedly, Chatzikyriakidis (2014) proposed the following that captures the aforementioned property:

(106) $\llbracket$ allegedly $\rrbracket=\lambda P:$ Prop. $\exists p: \llbracket$ human $\rrbracket, B_{p}(P)$

One of the advantages of this type of approach is that typing between intensional and non-intensional adjectives remains the same, i.e. no indices are needed in the typing. Thus, one can get away with the unwanted consequences of including intensional typing for the adjectives that are not intensional, a well known problem for Montagovian analyses of adverbs. Another welcome result of this line of approach, is that what have been dubbed as domain adverbs, can also easily be dealt with. Let

27 The same ideas discussed in the case of unknown beliefs in Sect. 3.2.1 are also relevant for unknown intentions. 
us explain. Assume a domain adjective like botanically or mathematically. Note that this is based on the assumption that domain adverbs are not veridical, i.e. that they do not presuppose the truth of the proposition they modify. ${ }^{28}$ Thus, in a sentence like botanically, tomato is a fruit, it does not follow that tomato is a fruit. This basically says, that tomato is a fruit in the context of botanology. The idea is to use contexts again in order to represent the domain in each case, i.e. the context of botanology (a collection of facts pertaining to botanology), using contexts. Thus, one can use the following for the adverb botanically and in general for domain adverbs:

(107) $\llbracket$ botanically $=\lambda P: \operatorname{Prop} . \Gamma_{B} P$

\subsection{Manner Adverbials}

Manner adverbials are a subcategory of predicational adverbs. They are VP adverbials and constrain the way/manner the subject performs the action denoted by the VP. Classic treatments of manner adverbs usually rely on Davidsonian assumptions according to which some sort of event modification is at play. For example, an adverb like slowly will specify that the event under consideration is a slow event. However, this does not really capture what we want, because it is the manner of the event rather than the event itself that is slow. These considerations have led researchers to argue for the inclusion of manners in their semantic ontology as well (Dik 1975; Schäfer 2008). For example Schafer proposes the following for the sentence John wrote illegibly:

(108) $\exists e[\operatorname{subject}(j o h n, e) \wedge$ write $(e) \wedge \exists m[$ manner $(m, e) \wedge$ illegible $(e)]]$

So how are we going to treat these type of adverbs in MTTs? The first thing to look at concerns the ontology. How are manners to be represented, if at all. The reader will remember our treatment of adjectives where the use of indexed $\mathrm{CN}$ types was proposed, in effect CNs indexed with degree parameters. The difference here is that modification is not about individuals but rather events. However, the idea to use indexed types seems promising in this case as well. Let us explain. First, we make the assumption that verbs subcategorize for an event argument, i.e. verbs also involve an event argument. We introduce the type Event:Type. Now assuming that a predicate will have to be of type $A \rightarrow$ Event $\rightarrow$ Prop (with $A: \mathrm{CN}$ ), a predicate modifier like e.g. a manner adverb should be of the following type:

$(109) \llbracket A D V_{V P} \rrbracket: \Pi A: \mathrm{CN} .(A \rightarrow$ Event $\rightarrow$ Prop $) \rightarrow(A \rightarrow$ Event $\rightarrow$ Prop $)$

Of course, in order for proper event semantics to be given events need to be structured. However, this is not the scope of this paper. Here we focus on how events can be modified by manner adverbs and the idea we want to put forward is that events just like $\mathrm{CNs}$ can be also indexed. We can introduce a general primitive type Manner:Type. With this we can assume the family of types Event:Manner $\rightarrow$ Prop indexed by

\footnotetext{
28 We will not get into a discussion on whether this is absolutely correct and some people might have different judgments with respect to this.
} 
manners, Event $(m)$ (with $m$ :Manner) is then the type of events of manner $m$. In sentences with no manner adverbs or in which there is no explicit mention on what the quality of the manner is, we can assume a contextually realized manner quality $R:$ Manner $\rightarrow$ Prop which corresponds to some kind of default manner quality depending on context. In the case of manner adverbials or similar expressions, the quality of the manner is explicitly specified. Thus for illegibly the relevant specification would be illegible $(m)$ etc. The lexical entry for illegibly will be as follows:

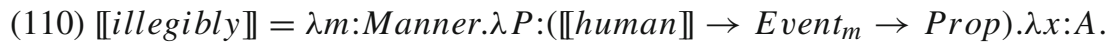 $\lambda E:$ Event $_{m} . P(x)(E) \wedge$ illeggible $(m)$}

Needless to say that the associated veridical inference is captured with the above entry, given that it is included as the first member of the conjunction. In effect, with this entry he wrote always follows from he wrote illegibly.

\subsection{Some Notes on Other Classes of Adverbs}

In this section we discuss some further issues relating to adverbial modification, outlining ways in which MTT-semantics can work towards an account. A natural category of adverbs to discuss, given the context of this paper, is subject oriented adverbs, or better agent oriented adverbs given that these involve the agent and not the subject. ${ }^{29}$ Such adverbs are traditionally looked at on a par with manner adverbs, given the existence of ambiguous readings, i.e. manner/agent-oriented, with a number of adjectives. Crucially, the accounts put forth assume a property of the subject on the basis of the truth of the proposition that the sentence without the adverb denotes. Thus, the sentence John stupidly called Mary means that John's act of calling Mary was stupid. In the literature, one finds accounts like McConnell-Ginet (1982) where manner adverbs are treated as arguments of the verb and agent oriented adverbs as predicate modifiers. Event based accounts treat agent oriented adverbs to involve some additional structure. For example, Rexach (1997) mentions that a way to capture the difference between the two classes is to assume that, in manner adverbs, the adjective is the event only, while in the case of agent oriented adverbs, both the event and the participant are:

$$
\text { (111) } \begin{aligned}
A D V_{\text {manner }} & =\lambda P \lambda x \exists e . P(e, x) \wedge A d j_{A D V}(e) \\
A D V_{\text {agent }} & =\lambda P \lambda x \exists e . P(e, x) \wedge A d j_{A D V}(e, x)
\end{aligned}
$$

A solution in MTT-semantics, that maintains the core of the analysis of manner adverbs, will involve again indexed types. But now, instead of the type of events indexed only by manners, what we have is types of events which are indexed by humans as well as manners. Thus, 【human $(m) \rrbracket$ is the type of humans with manner $m$.

\footnotetext{
${ }^{29}$ Evidence from passive constructions shows that this is the case. Thus, in the boat was sunk intentionally by the government, the paraphrase we get is that it was intentional on behalf of the government to sink the boat, rather than it was intentional on behalf of the boat to sink itself that a subject oriented interpretation would imply. See Jackendoff (1972), McConnell-Ginet (1982), Geuder (2000) for more details on agent oriented adverbs.
} 
The semantics of speaker oriented adverbs seem more difficult to grasp. Here, we are going to only look at speech act adverbials like honestly, frankly. Such adverbs can be seen as providing commentary with respect to the utterance. In this respect, the sentence Frankly, I do not know what to say, roughly means I frankly tell you that I do not know what to say. This paraphrase dating back to Schreiber (1972) gives rise to a way of looking at speech act adverbs that is not that different from manner adverbs. Piñón (2013) provides an interesting account according to which speaker oriented adverbs make reference to individual manners of speaking. We will not go into the details of his proposal. Assuming that this be a reasonable way to look at speaker oriented adverbials, one can sketch an account in MTTs as follows.

First, the type of utterance events are indexed by utterers and manners:

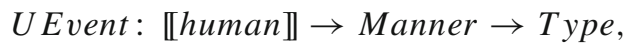

that is, $U$ Event $(u, m)$ is the type of events with utterer $u$ and manner $m$. For example, the type of frank can be given as follows:

$$
\llbracket \text { frank } \rrbracket: \Pi u: \text { Utterer. Пm: Manner. UEvent }(u, m) \rightarrow \text { Prop. }
$$

Then, the adverb frankly can now be given the following definition: for any $u$ : Utterer, $m$ : Manner and $e: U \operatorname{Event}(u, m)$,

$$
\llbracket f r a n k l y \rrbracket(u, m, e)=_{\mathrm{df}} \lambda P: \operatorname{Prop} . P \wedge \operatorname{frank}(u, m, e)
$$

and frankly thus defined is of the following type:

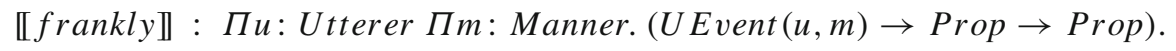

In fact, all of such agent-oriented adverbs can be defined as above.

According to the account just presented, a speech act adverb is not of type Prop $\rightarrow$ Prop; instead it takes both an utterance event as well as a proposition as arguments and returns a proposition.

With this last remark, we will stop our discussion on adverbial modification, leaving a number of issues unresolved. To recap, we have shown that MTTs can provide us with a rich and expressive typed language in order to deal with a number of aspects pertaining to modification. From a theoretical point of view, we hope that we have presented arguments for using MTTs for NL semantics.

\section{Modification and Inference}

One attractive characteristic of MTT-semantics is that it can be seen as proof-theoretic (Luo 2014). This means that the judgments in the underlying type theories can be understood by means of their inferential roles. This latter fact constitutes MTTs a good solution w.r.t consequences that the semantics proposed in each case bring about, i.e. inference. This proof-theoretic aspect of MTTs has been the reason that these are 
widely implemented in computer reasoning systems, i.e. proof assistants. Proof assistant technology has gone a long way since its emergence. The proof-assistant Coq is a prime example of the advance reached in the field and a number of remarkable developments have been achieved via its use [e.g. see the proof for the four colour theorem (Gonthier 2005)]. Coq implements the Calculus of Inductive Constructions (CiC), in effect an MTT. Actually, CiC is quite close to the MTT we are using, i.e. UTT with coercive subtyping (Luo 1994; Luo et al. 2012). The fact that Coq 'speaks' so to say an MTT, in combination with the fact that it is a powerful reasoning engine, makes Coq suitable to implement and further reason about MTT semantics. The authors have exemplified the use of Coq as a means to deal with NLI in various papers (Chatzikyriakidis and Luo 2014b, a, 2016). There, it was shown that Coq can be used as a NL reasoner formalizing part of the FraCaS test suite examples and reasoning about them. Besides the various practical/computational applications that such an endeavour can lead to, there is an additional side to using Coq, which has to do with the correctness of the accounts one proposes. A correct account of a certain NL phenomenon should be able to derive all the correct consequences associated with it, while on the other hand it should not derive any unwanted consequences. This is basically to say that a correct account is judged by the number of inferences it gives rise to as well as the number of them that it does not. In our case and given the nice interaction of MTT-semantics and the associated proof-technology, we can actually check whether what the propose derives the proper inferences or not. This is what we are going to see now, by looking into the consequences that a number of our proposals made in this paper give rise to. In what follows we give a short introduction to Coq and then test the predictions of our account using MTT derivations for some cases and also presenting the relevant implementations in Coq along with their explanation in the "Appendix".

\subsection{The Coq Proof Assistant}

The idea behind Coq is simple and can be roughly summarized as follows: you use Coq in order to see whether propositions based on statements previously pre-defined or user defined (definitions, parameters, variables) can be proven or not. As we have said, is a dependently typed proof-assistant implementing the calculus of Inductive Constructions (CiC, see Coq 2007). This means that the language used for expressing these various propositions is an MTT. To give a very short example of how Coq operates let us try to prove a propositional tautology in the system, let us say the following:

$$
((P \vee Q) \wedge(P \rightarrow R) \wedge(Q \rightarrow R)) \rightarrow R
$$

Given Coq's typed nature we have to introduce the variables $P, Q, R$ as being of type Prop ( $P, Q, R:$ Prop). Now in order to get Coq into proof mode, we have to use the command Theorem followed by the name we give to this theorem and then followed by the theorem we want to prove:

(113) Theorem $A:((P \vee Q) \wedge(P \rightarrow R) \wedge(Q \rightarrow R)) \rightarrow R$ 
This will put Coq into proof mode:

Theorem $A:((P \backslash / Q) /(P->R) / \backslash(Q->R))->R$.

1 subgoal

$===$ = = = = = = = = = = = = = = = = =

$(\mathrm{P} \backslash / \mathrm{Q}) / \backslash(\mathrm{P}->\mathrm{R}) / \backslash(\mathrm{Q}->\mathrm{R})->\mathrm{R}$

Now, we have to guide the prover to a proof using its pre-defined proof tactics (or we can define our own). For the case interested, we first introduce the antecedent as an assumption using intro:

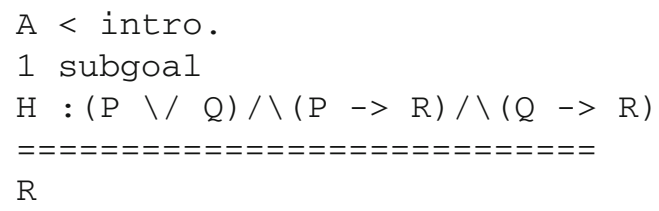

We split the hypothesis into individual hypotheses using destruct destruct HO.

1 subgoal

$\mathrm{H}: \mathrm{P} \backslash / \mathrm{Q}$

$\mathrm{HO}: \mathrm{P} \rightarrow \mathrm{R}$

$\mathrm{H} 1$ : $\mathrm{Q} \rightarrow \mathrm{R}$

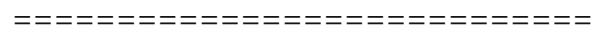

$\mathrm{R}$

Now, we can apply the elimination rule for disjunction which will basically result in two subgoals:

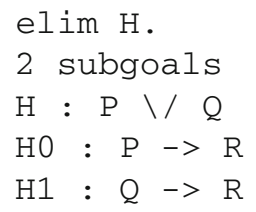

The two subgoals are already in the hypotheses. We can use the assumption tactic that matches the goal with an identical hypothesis and the proof is completed:

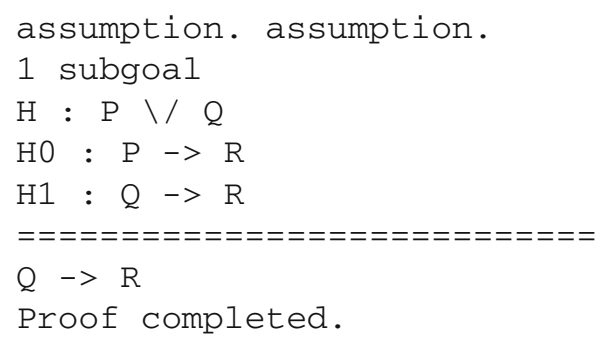


Now, in order to reason with NL semantics, we basically implement our theoretical work on NL semantics and then look at the consequences these have as regards inference. To give an example, consider the case of the existential quantifier $a$. Quantifiers in MTTs are given the following type, where $A$ extends over the $\mathrm{CN}$ (this is reminiscent of the type used for VP adverbs):

(114) $\Pi$ A: CN. $(A \rightarrow$ Prop $) \rightarrow(A \rightarrow$ Prop $)$

We provide a definition based on this type, giving rather standard semantics for the existential (in Coq notation):

Definition some:=fun $(\mathrm{A}: \mathrm{CN})(\mathrm{P}: \mathrm{A}->$ Prop $)=$ exists $\mathrm{x}: \mathrm{A}, \mathrm{P} \mathrm{x}$.

This says that given an $A$ of type $\mathrm{CN}$ and a predicate over $A$, there is an $x: A$ such that $P$ holds of $x$.

Imagine that we want to see the consequences of this definition. For example we may want to check whether John walks implies that some man walks or that some man walks implies that some human walks. We define, following our theoretical assumptions about $\mathrm{CNs}$, man and human to be of type $\mathrm{CN}$ and declare the subtyping relation $\llbracket m a n \rrbracket<\llbracket h u m a n \rrbracket$. This is all we need to get the above inferences. These assumptions suffice to prove these inferences in Coq.

\subsection{Testing the Theory}

In this section we check a number of the accounts we have provided with respect to the inferences they give or do not give rise to. At first let us see the inferences we get with respect to intersective adjectives. Traditionally, the inferences of intersective adjectives that need to be captured are the following two: ${ }^{30}$

(115) $\operatorname{Adj}(N)(x) \Rightarrow N(x)$.

(116) $\operatorname{Adj}\left(\mathrm{N}_{1}\right)(\mathrm{x}) \Rightarrow \mathrm{N}_{1}(\mathrm{x}) \wedge \operatorname{Adj}\left(\mathrm{N}_{2}\right)(\mathrm{x})$, if $\mathrm{N}_{1}<\mathrm{N}_{2}$.

The first inference is taken care of by the $\Sigma$ type analysis, where the first projection is declared as a coercion. We exemplify this with the example showing a man walks

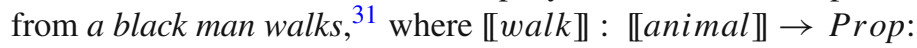

- $\Sigma($ man, black $)<\llbracket m a n \rrbracket<\llbracket$ animal $\rrbracket$ (by first projection as coercion);

- therefore, $\exists x: \Sigma$ (man, black). walk(x) implies $\exists y:$ man. walk $(y)$;

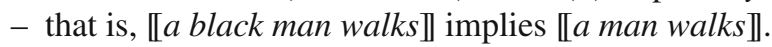

The second inference can be done in a similar way, given the rules for coercive subtyping as in Luo (1997), Luo et al. (2012). For example, we can infer a black human walks from a black man walks.

\footnotetext{
30 Again, note that in MTTs, $N(x)$ will be the type judgment, $x: N$.

31 This is easily proven in Coq (we use dependent record types for encoding the $\Sigma$ type approach). See "Appendix" for the actual code and example.
} 
However, encoding this in Coq is trickier. This has to do with a defect in Coq's coercive subtyping mechanism. In a nutshell, Coq does support subtyping, but however does not allow subtyping to propagate through the constructors, i.e. in our case it does not allow the inference $\Sigma(\llbracket$ man $\rrbracket, \llbracket$ black $\rrbracket)<\Sigma(\llbracket$ human $\rrbracket, \llbracket$ black $\rrbracket)$ given $\llbracket m a n \rrbracket<\llbracket h u m a n \rrbracket .^{32}$ Thus, one has to declare individual coercions between the Sigma types, e.g. $\Sigma(\llbracket m a n \rrbracket, \llbracket b l a c k \rrbracket)<\Sigma(\llbracket h u m a n \rrbracket, \llbracket b l a c k \rrbracket)$. However, once we do that an additional problem arises: that of ambiguous paths. Given that $\Sigma(\llbracket$ man $\rrbracket, \llbracket$ black $\rrbracket)<\llbracket$ man $\rrbracket, \Sigma(\llbracket h$ hman $\rrbracket, \llbracket$ black $\rrbracket)<\llbracket$ human $\backslash$ there are two paths from $\Sigma(\llbracket m a n \rrbracket, \llbracket b l a c k \rrbracket)$ to $\llbracket h u m a n \rrbracket$, one via $\Sigma(\llbracket m a n \rrbracket, \llbracket b l a c k \rrbracket)<$ $\llbracket m a n \rrbracket<\llbracket$ human $\rrbracket$, and one via $\Sigma(\llbracket$ man $\rrbracket, \llbracket$ black $\rrbracket)<\Sigma(\llbracket$ human $\rrbracket, \llbracket$ black $\rrbracket)<$

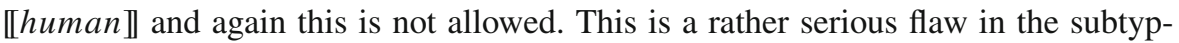
ing mechanism that does not allow to evaluate our account at this point. Note that the proof-assistant Plastic that implements UTT does not give rise to this problem. In order to sidestep this, we basically introduce a hack, according to which black man has two senses: on one sense, it is associated with a coercion to man, while on the other, with a coercion to black human. If we do this, the inference we want goes through and we can prove things like the following: ${ }^{33}$

(117) A black man walks $\Rightarrow$ A black human walks

Note that this problem of inference that had to be sidestepped in this way is only present in the attributive use of the adjective, i.e. when a $\Sigma$ type is involved. In predicational uses, this problem in inference does not arise and examples like the following are predicted without any hacks in the Coq code: ${ }^{34}$

(118) Some man is black $\Rightarrow$ Some human is black

Turning to subsective adjectives, we need to capture the fact that:

(119) from $\operatorname{Adj}(\mathrm{N})$ infer $\mathrm{N}$.

The polymorphic type we proposed for adjectives really takes care of this, since it will assume that the inference is valid for the specific class in each case. The inference from $\operatorname{Adj}(\mathrm{N})$ is captured via the first projection coercion, no surprises here. Now, one will want to see that an inference of the following sort is not valid for subsective adjectives but it is true for intersective adjectives:

some $\mathrm{x} \operatorname{Adj} \Rightarrow$ some y Adj (where $x<y)$

Indeed, for intersective adjectives, this is the case. For example assuming black is of type object $\rightarrow$ Prop, the above can be proven. However, given the typing we have proposed for subsective adjectives, no proof is found. For if we try to prove George is

\footnotetext{
32 Such propagations of suutyping relations through type constructors is desirable, but it is not implemented in Coq.

33 See "Appendix" for the actual code.

34 See "Appendix" for the code and example
} 
a small animal from George is a small man we are stuck, since we are basically trying to prove small(animal)(George) from small(man)(George). Given that small is relativized to different domains in each case, it seems that no proof can be found.

With privative adjectives like fake, and we assume an analysis as this was sketched in this paper, where these involve coercion of the $\mathrm{CN}$ to include fake $\mathrm{CN}$ denotations, we proposed a disjoint union type. Using this type we can predict that a fake gun is a gun but it is not a real gun. For example one can prove that all real guns are not fake guns and vice versa in Coq

The account put forth for degree adjectives has already been checked using Coq in Chatzikyriakidis and Luo (2014b) for the comparative cases. It is rather straightforward to extend it to the positive case. We will not do it here for reasons of space. We will rather look at the case of multidimensional adjectives. The account proposed here can be straightforwardly encoded in Coq by defining an inductive type Health in the same sense we did in $81: 35$

Inductive Health: CN:=Heart|Blood_pressure|Cholesterol|...

Then following the ideas sketched in this paper for multidimensional adjectives one can derive the following in Coq (see "Appendix" for the code):

(121)John is sick $\Rightarrow$ John is not healthy

(122)John is not healthy with respect to one health dimension $\Rightarrow$ John is sick

(123)John is healthy with respect to one health dimension $\nRightarrow$ John is healthy

Similarly for adverbs, we can also check the accounts proposed. We show this by giving the example of veridical VP adverbs. For example, if we define slowly as in (90), the inferences like the one shown below follow:

(124) John walks slowly $\Rightarrow$ John walks

since, by (90), one obtains from the second projection that walk slowly implies walk.

As an additional example, consider the analysis we have provided for manner adverbs in this paper. We can implement this in Coq. By doing so, we can get inferences like:

(125) John writes illegibly $\Rightarrow$ John wrote in an illegible manner.

We have not yet tried the intensional cases of adverbs. We leave this as future work, even though we believe that this will not be difficult to do. In particular, Coq's Local section mechanism (in effect local contexts) would be useful for implementing the account of domain adverbials. But as we have said, we end the discussion here, leaving these issues for future research.

\footnotetext{
35 The '...' is not part of the actual code. It just says that more health dimensions can be added depending on the fine-grainedness we want to achieve.
} 
Open Access This article is distributed under the terms of the Creative Commons Attribution 4.0 International License (http://creativecommons.org/licenses/by/4.0/), which permits unrestricted use, distribution, and reproduction in any medium, provided you give appropriate credit to the original author(s) and the source, provide a link to the Creative Commons license, and indicate if changes were made.

\section{Appendix: The Examples in Coq}

The first case involves the inference a black man $\Rightarrow$ a black human.

The following are needed:

Definition

$\mathrm{CN}$ :=Set. Parameter Man Human Object: CN *declaring the base types* Axiom mh:Man->Human. Coercion mh:Man >-> Human. *subtyping* Axiom ho:Human->object. Coercion ho:Human>-> Object. *subtyping* Definition some:= fun $\mathrm{A}: \mathrm{CN}$, fun $\mathrm{P}: \mathrm{A}->$ Prop $=>$ exists $\mathrm{x}: \mathrm{A}, \mathrm{P}(\mathrm{x})$. *quantifier* Parameter black:object->Prop.

What we want to prove is:

1 subgoal

=ニ=ニ= = = = = = = = = = = = = = =

some Man (fun $\mathrm{x}$ : Man $=>$ black $\mathrm{x}$ ) $->$ some Human (fun $\mathrm{x}$ : Human $=>$ black $\mathrm{x}$ )

Unfolding the definition for the quantifier and using intro, we move the antecedent as a premise:

$\mathrm{H}$ : exists $\mathrm{x}$ : Man, black $\mathrm{x}$

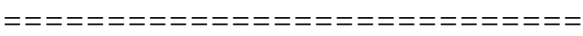

exists $\mathrm{x}$ : Human, black $\mathrm{x}$

Then, the destruct tactic first performs elim, it finds the appropriate destructor, applies it and then uses intro. The result is:

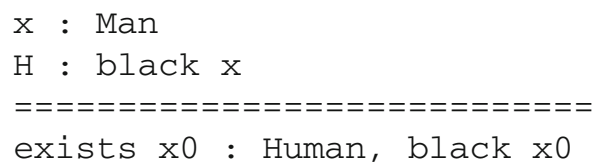

What we need to do is substitute $x$ for $x 0$. Applying assumption which matches a goal with a premise finishes the proof. However, as we have said for the attributive case, we need a hack, since in Coq, subtyping does not propagate through the constructors. So in order to prove a black human walks from a black man walks some additional coding is needed as explained in 6.2:

Record Blackman:CN:=mkBlackman $\{14:>$ Man; _ :Black 14$\}$.

Record Blackhuman:CN:=mkBlackhuman $\{15:>$ Human; _ :Black 15$\}$.

Record Blackman 1:CN:=mkBlackman1\{c4:>Blackhuman; _ :Black c4 $\}$.

Inductive OneBlackman:Set:=BlackMan .

Definition Br1 ( $r$ :OneBlackman) $: \mathrm{CN}:=\mathrm{Bl}$ ackman. Coercion Br1:OneBlackman $>->\mathrm{CN}$.

Definition Br2 ( $r$ :OneBlackman): Set:=Blackman1. Coercion Br2:OneBlackman>->Sortclass .

Moving on to subsective adjectives, we want to check whether we can infer George is a large animal from George is a large man. First the code needed: 
Parameter

Animal:CN *declaring the base types* Axiom ha:Human->Animal.

Coercion ha:Human >-> Animal. *subtyping* Axiom ao: Animal->object. Coercion ao:Animal >->object. *subtyping* Parameter George:Man. Parameter large:forall A:CN, A -> Prop. *polymorphic type*

We formulate the example:

1 subgoal

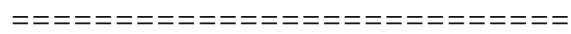

Large Man George -> Large Animal George

We introduce the antecedent as a premise. But it seems that there is nothing we can do besides that:

1 subgoal $\mathrm{H}$ : Large Man George

$======================$ =

Large Animal George

The polymorphic nature of Large does not seem to allow the inference to go through.

As regards inference with fake, the following are needed:

Parameter Gun_r:CN.

Parameter Gun_f:CN.

Definition GUN:= (sum Gun_r Gun_f).

Axiom gun1:Gun_r-> GUN. Coercion gun1: Gun_r>-> GUN .

Axiom gun2:Gun_f-> GUN. Coercion gun2: Gun_f>->GUN .

Fixpoint fake $(\mathrm{A}: \mathrm{CN}) \quad(\mathrm{x}: \mathrm{GUN}):=$ match $\mathrm{x}$ with $\mid$ inl _ => False | inr _ => True end.

Fixpoint real $(\mathrm{A}: \mathrm{CN}) \quad(\mathrm{x}: \mathrm{GUN}):=$ match $\mathrm{x}$ with $\mid$ inl _ => True | inr _ => False end.

With these, we can prove that all guns, if they are real they are not fake and vice versa. We first use cbv which applies all reduction rules possible and then intuition, which finds all first order intuitionistic logic tautologies:

Coq < Theorem GUN1: forall fr: GUN, real GUN fr<-> not (fake GUN fr).

GUN1 < cbv. intuition.

1 subgoal

Proof completed.

For inference with veridical adverbs like slowly, we need the following:

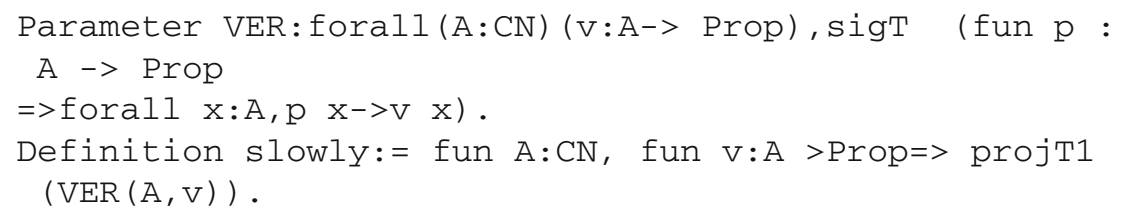


Let us say we want to prove that John walks from John walks slowly:

1 subgoal

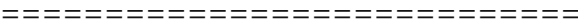
slowly walk John $->$ walk John

We unfold the definitions and use destruct for the auxiliary object:

1 subgoal

$\mathrm{x}$ : Animal $->$ Prop

$\mathrm{w}$ : forall $\mathrm{x} 0$ : Animal, $\mathrm{x} \times 0->$ walk $\mathrm{x} 0$

$======================$

$\mathrm{x}$ John $->$ walk John

We use apply $w$ and the proof is completed.

Lastly, for multidimensional adjectives, we provide the code only. The reader is encouraged to try the examples.

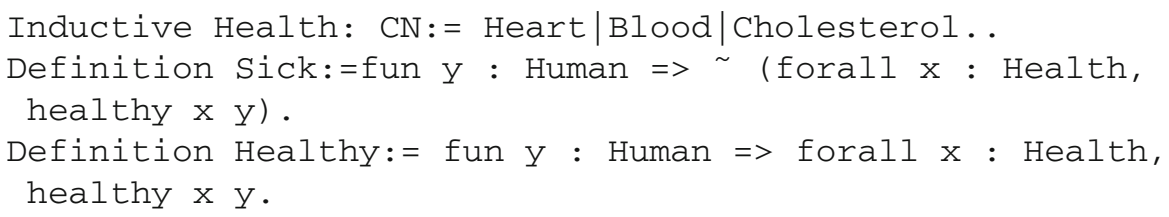

The same goes for the inferences regarding manner adverbs:

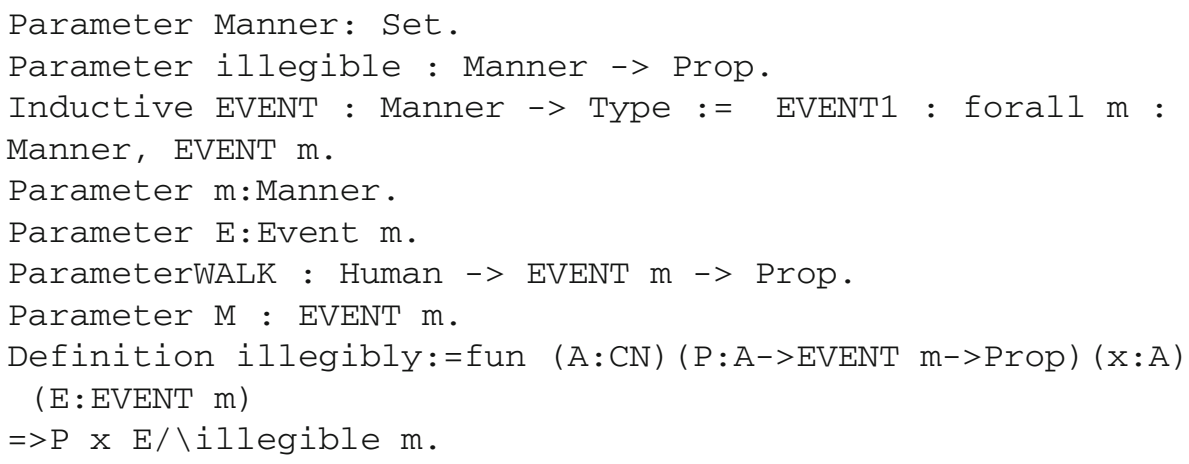

\section{References}

Asher, N. (2012). Lexical meaning in context: A web of words. Cambridge: Cambridge University Press.

Asher, N. \& Luo, Z. (2012). Formalisation of coercions in lexical semantics. Sinn und Bedeutung 17, Paris, 223

Bartsch, R., \& Vennemann, T. (1973). Semantic structures: A study in the relation between syntax and semantics. Frankfurt: Athaeenum Verlag.

Boldini, P. (2000). Formalizing contexts in intuitionistic type theory. Fundamenta Informaticae, 4(2)

Boldini, P. (2001). The reference of mass terms from a type-theoretical point of view. In Proceedings of the 4th International Workshop on Computational Semantics. Tilburg, Netherlands.

Chatzikyriakidis, S. (2014). Adverbs in a modern type theory. In N. Asher \& S. Soloviev (Eds.), Proceedings of LACL2014. LNCS 8535 (pp. 44-56) 
Chatzikyriakidis, S. \& Luo, Z. (2013). Adjectives in a modern type-theoretical setting. In G. Morrill \& J. M. Nederhof (Eds.), Proceedings of formal grammar 2013. LNCS 8036 (pp. 159-174)

Chatzikyriakidis, S. \& Luo, Z. (2014a). Natural language reasoning using proof-assistant technology: Rich typing and beyond. In Proceedings of EACL2014

Chatzikyriakidis, S., \& Luo, Z. (2014b). Natural language inference in Coq. Journal of Logic, Language and Information, 23(4), 441-480.

Chatzikyriakidis, S. \& Luo, Z. (2014c). Using signatures in type theory to represent situations. Logic and Engineering of Natural Language Semantics 11. Tokyo

Chatzikyriakidis, S. \& Luo, Z. (2016). Proof assistants for natural language semantics. In M. Amblard, P. de Groote, S. Pogodalla \& C. Retoré (Eds.), Logical aspects of computational linguistics. Celebrating 20 years of $L A C L$ (pp. 85-98)

Church, A. (1940). A formulation of the simple theory of types. The Journal of Symbolic Logic, 5(1), 56-68.

Coppock, E. (2008). The logical and empirical foundations of Baker's Paradox. ProQuest

Coq 2007. (2007). The Coq Proof Assistant Reference Manual (Version 8.1), INRIA. The Coq Development Team.

Davidson, D. (1967). Compositionality and coercion in semantics: The semantics of adjective meaning. In N. Rescher (Ed.), The logical form of action sentences (pp. 81-95). Pittsburgh: University of pittsburgh Press.

Dik, S. (1975). The semantic representation of manner adverbials. In A. Kraak (Ed.), Linguistics in the Netherlands (pp. 1972-1973). Assen: Van Gorcum.

Dowty, D. R. (1981). Introduction to montague semantics. Berlin: Springer.

Enc, M. (1981). Tense without scope: An analysis of nouns as indexicals. PhD thesis, University of Wisconsin-Madison

Ernst, T. B. (2002). The syntax of adjuncts (Vol. 96). Cambridge: Cambridge University Press.

Geuder, W. (2000). Oriented adverbs: Issues in the lexical semantics of event adverbs.

Girard, J.-Y. (1971). Une extension de l'interpretation fonctionelle de gödel à l'analyse et son application à l'élimination des coupures dans et la thèorie des types'. In Proceedings of 2 nd scandinavian logic symposium. North-Holland

Gonthier, G. (2005). A computer-checked proof of the four-colour theorem. http://research.microsoft.com/ qgonthier/4colproof.pdf.

Heim, I. (2000a). Degree operators and scope. In Proceedings of SALT (Vol. 10, pp. 40-64).

Heim, I. (2000b). Degree operators and scope. In Proceedings of SALT (Vol. 10, pp. 40-64).

Jackendoff, R. (1972). Semantic interpretation in generative grammar. Cambridge: MIT Press.

Kahle, R. \& Schroeder-Heister, P. (Eds.) (2006). Proof-theoretic semantics. Special Issue of Synthese (Vol. 148 , No. 3).

Kamp, H. (1975). Formal semantics of natural language. In E. Keenan (Ed.), Two theories about adjectives (pp. 123-155). Cambridge: cambridge University Press.

Kamp, H., \& Partee, B. (1995). Prototype theory and compositionality. Cognition, 57(2), 129-191.

Kennedy, C. (1999). Projecting the adjective: The syntax and semantics of gradability and comparison. New York: Routledge.

Klein, E. (1980). A semantics for positive and comparative adjectives. Linguistics and Philosophy, 4(1), $1-45$.

Kratzer, A. (1989). An investigation of the lumps of thought. Linguistics and Philosophy, 12(5), 607-653.

Lassiter, D. (2014). Adjectival modification and gradation. In S. Lappin \& C. Fox (Eds.), Wiley-Blackwell handbook of contemporary semantics. New York: Blackwell.

Lewis, D. (1970). General semantics. Synthese, 22, 18-67.

Luo, Z. (1994). Computation and reasoning: A type theory for computer science. New York: Oxford University Press.

Luo, Z. (1997). Coercive subtyping in type theory. In CSL'96, LNCS'1258

Luo, Z. (1999). Coercive subtyping. Journal of Logic and Computation, 9(1), 105-130.

Luo, Z. (2010). Type-theoretical semantics with coercive subtyping. Semantics and linguistic theory 20 (SALT20), Vancouver

Luo, Z. (2011a). Adjectives and adverbs in type-theoretical semantics. Notes

Luo, Z. (2011b). Contextual analysis of word meanings in type-theoretical semantics. In Logical aspects of computational linguistics (LACL'2011). LNAI 6736.

Luo, Z. (2012a). Common nouns as types. In D. Bechet \& A. Dikovsky (Eds.), Logical aspects of computational linguistics (LACL'2012). LNCS 7351. 
Luo, Z. (2012b). Formal semantics in modern type theories with coercive subtyping. Linguistics and Philosophy, 35(6), 491-513.

Luo, Z. (2014). Formal semantics in modern type theories: Is it model-theoretic, proof-theoretic, or both? Invited talk at logical aspects of computational linguistics 2014 (LACL 2014), Toulouse. In LNCS (Vol. 8535, pp. 177-188).

Luo, Z., Soloviev, S., \& Xue, T. (2012). Coercive subtyping: Theory and implementation. Information and Computation, 223, 18-42.

Maienborn, C., \& Schafer, M. (2011). Adverbs and adverbials. In C. Maienborn, K. von Heusinger, \& P. Portner (Eds.), Semantics. An international handbook of natural language meaning (pp. 1390-1420). Mouton: De Gruyter.

Martin-Löf, P. (1975). An intuitionistic theory of types: Predicative part. In H. Rose \& J. C. Shepherdson (Eds.), Logic Colloquium'73

Martin-Löf, P. (1984). Intuitionistic type theory. Napoli: Bibliopolis.

McConnell-Ginet, S. (1982). Adverbs and logical form: A linguistically realistic theory. In Language (pp. 144-184).

McConnell-Ginet, S. M. (1973). Comparative constructions in English: A syntactic and semantic analysis.

Montague, R. (1970). English as a formal language. In B. Visentini (Ed.), Linguaggi nella Societ e nella. Milan: Edizioni di Comunit, 1.

Montague, R. (1973). The proper treatment of quantification in ordinary English. In J. Hintikka, J. Moravcsik \& P. Suppes (Eds.), Approaches to natural languages.

Montague, R. (1974). Formal philosophy. Yale University Press, Collected papers edited by R. Thomason.

Morzycki, M. (2014). Modification to appear in the series series key topics in semantics and pragmatics. Cambridge: Cambridge University Press.

Musan, R. I. (1995). On the temporal interpretation of noun phrases. PhD thesis, Massachusetts Institute of Technology.

Nordström, B., Petersson, K., \& Smith, J. (1990). Programming in Martin-Löf's type theory: An introduction. Oxford: Oxford University Press.

Nunberg, G. (1995). Trasfers of meaning. Journal of Semantics, 12(2), 109-132.

Partee, B. (2007) ompositionality and coercion in semantics The semantics of adjective meaning. In G. Bouma, I. M. Krämer, \& J. Zwarts (Eds.), Cognitive foundations of interpretation (pp. 145-161). Royal Netherlands Academy of Arts and Sciences.

Partee, B. (2010). Privative adjectives: Subsective plus coercion. In R. Bauerle \& U. Reyle (Eds.), Presuppositions and discourse: Essays offered to Hans Kamp (pp. 123-155). Emerald Group Publishing.

Pierce, B. (1991). Programming with intersection types, union types, and polymorphism. Technical report CMU-CS-91-106, School of Computer Science, CMU.

Piñón, C. (2013). Speech-act adverbs as manner adverbs. Ms. Univesity of Lille.

Ranta, A. (1994). Type-theoretical grammar. Oxford: Oxford University Press.

Rexach, J. G. (1997). Questions and generalized quantifiers. Berlin: Springer.

Sassoon, G. W. (2012). A typology of multidimensional adjectives. Journal of Semantics, 30(3), 335-380.

Schäfer, M. (2008). Resolving scope in manner modification. Empirical Issues in Syntax and Semantics, 7 , 351-372.

Schreiber, P. A. (1972). Style disjuncts and the performative analysis. In Linguistic inquiry (pp. 321-347). MIT press.

Sundholm, G. (1989). Constructive generalized quantifiers. Synthese, 79(1), 1-12.

Thomason, R. H., \& Stalkaner, R. C. (1973). A semantic theory of adverbs. Linguistic Inquiry, 4(2), 195220.

Tonhauser, J. (2002). A dynamic semantic account of the temporal interpretation of noun phrases. In Semantics and linguistic theory (pp. 286-305)

Van Benthem, J. (2012). The logic of time: A model-theoretic investigation into the varieties of temporal ontology and temporal discourse (Vol. 156). Berlin: Springer.

Von Stechow, A. (1984). Comparing semantic theories of comparison. Journal of Semantics, 3(1), 1-77. 